\title{
ZS Research Square

\section{Integrated analysis of cervical squamous cell carcinoma cohorts from three continents reveals conserved subtypes of prognostic significance.}

\section{Ankur Chakravarthy}

Princess Margaret Cancer Centre

lan Reddin

University of Southampton

\section{Stephen Henderson}

UCL Cancer Institute https://orcid.org/0000-0002-9032-3828

Cindy Dong

University of Kent

Nerissa Kirkwood

School of Biosciences, University of Kent

Maxmilan Jeyakumar

School of Biosciences, University of Kent

Daniela Rothschild-Rodriguez

University of Kent

Natalia Gonzalez-Martinez

University of Kent https://orcid.org/0000-0003-1038-5306

Jacqueline McDermott

University College London

Xiaoping Su

Dan L. Duncan Cancer Center and, Baylor College of Medicine

Nagayasau Egawa

University of Cambridge

Christina Fjeldbo

Department of Radiation Biology, Oslo University Hospital

\section{Vilde Skingen}

Oslo University Hospital

\section{Mari Halle}

Haukeland Univesity Hospital

\section{Camilla Krakstad}

University of Bergen https://orcid.org/0000-0002-0174-8139 
INNPATH Institute of Pathology

\section{Susanne Sprung}

Institute of Pathology, Medical University of Innsbruck

\section{Peter Ellis}

University of Kent https://orcid.org/0000-0001-9709-7934

\section{Mark Wass}

University of Kent https://orcid.org/0000-0001-5428-6479

\section{Martin Michaelis}

University of Kent https://orcid.org/0000-0002-5710-5888

\section{Heidi Lyng}

Oslo University Hospital https://orcid.org/0000-0002-7938-0514

\section{Heidelinde Fiegl}

Medical University of Innsbruck, Innsbruck https://orcid.org/0000-0002-1236-6806

\section{Helga Salvesen}

Department of Obstetrics and Gynaecology, Haukeland University Hospital; Centre for Cancer Biomarkers, Department of Clinical Science, University of Bergen

\section{Gareth Thomas}

University of Southampton

\section{John Doorbar}

University of Cambridge

\section{Kerry Chester}

University College London

\section{Andrew Feber}

Royal Marsden Hospital

\section{Tim Fenton ( $\nabla$ t.fenton@soton.ac.uk)}

University of Southampton https://orcid.org/0000-0002-4737-8233

\section{Article}

\section{Keywords:}

Posted Date: December 22nd, 2021

DOI: https://doi.org/10.21203/rs.3.rs-1129380/v1

License: (c) (i) This work is licensed under a Creative Commons Attribution 4.0 International License. Read Full License

Version of Record: A version of this preprint was published at Nature Communications on October 7th, 2022. See the published version at https://doi.org/10.1038/s41467-022-33544-x. 


\section{Abstract}

Human papillomavirus (HPV)-associated cervical cancer represents one of the leading causes of cancer death worldwide. Although low-middle income countries are disproportionately affected, our knowledge of the disease predominantly originates from populations in high-income countries. Using the largest multi-omic analysis of cervical squamous cell carcinoma (CSCC) to date, totalling 643 tumours and representing patient populations from the USA, Europe and Sub-Saharan Africa, we identify two CSCC subtypes (C1 and $\mathrm{C} 2$ ) with differing prognosis. $\mathrm{C} 1$ tumours are largely HPV16-driven, display increased cytotoxic T-lymphocyte infiltration and frequently harbour PIK3CA and EP300 mutations. C2 tumours are associated with shorter overall survival, are frequently driven by HPVs from the HPV18-containing alpha-7 clade, harbour alterations in the Hippo signalling pathway and increased expression of immune checkpoint genes, B7-H3 (also known as CD276) and NT5E (also known as CD73) and PD-L2 (also known as $P D C D 1 L G 2$ ). In conclusion, we identify two novel, therapy-relevant CSCC subtypes that share the same defining characteristics across three geographically diverse cohorts.

\section{Full Text}

Despite screening and the introduction of prophylactic human papillomavirus (HPV) vaccination in developed countries, cervical cancer continues to be one of the leading worldwide causes of cancerrelated deaths in women ${ }^{1}$. Prognosis for patients with metastatic disease remains poor, thus new treatments and effective molecular markers for patient stratification are urgently required. Cervical cancer is caused by at least 14 high-risk human papillomaviruses (hrHPVs), with HPV16 and HPV18 together accounting for over $70 \%$ of cases worldwide, with some variation by region ${ }^{1,2}$. Cervical squamous cell carcinoma (CSCC) is the most common histological subtype of cervical cancer, accounting for approximately $60-70 \%$ of cases, again with some variation seen across different populations ${ }^{2}$. Adenoand adenosquamous histology are both associated with poor prognosis ${ }^{3-6}$, while the relationship, if any, between HPV type and cervical cancer prognosis remains unclear ${ }^{7}$. HPV type is also associated with histology, with HPV16 more commonly found in CSCC, while adenocarcinomas are more likely to harbour HPV182 ${ }^{2}$. Previous landmark studies described the genomic landscape of cervical cancer in different populations $^{8-11}$ and in some cases identified subtypes based on gene expression, DNA methylation and/or proteomic profiles ${ }^{8,9}$. The Cancer Genome Atlas (TCGA) network identified clusters based on RNA, micro-RNA, protein/phospho-protein, DNA copy number alterations and DNA methylation patterns and combined data from multiple platforms to define integrated iClusters ${ }^{8}$. In their analysis, only clustering based on the expression levels and/or phosphorylation state of 192 proteins as measured by reversephase protein array (RPPA) was associated with outcome, with significantly shorter overall survival (OS) observed for a cluster of cervical cancers exhibiting increased expression of Yes-associated protein (YAP) and features associated with epithelial-to-mesenchymal transition (EMT) and a reactive tumour stroma. Since TCGA's RPPA analysis was restricted to 155 tumours including SCCs, adeno- and adenosquamous carcinomas, we set out to test the hypothesis that with data from more samples, we could identify a set of transcriptional and epigenetic features associated with prognosis within CSCC and to establish 
whether it is also present in independent patient cohorts representing different geographical locations and ethnicities. To identify molecular subtypes and prognostic correlates, we identified a set of 643 CSCCs (all HPV-positive), for which clinico-pathological data and genome-wide DNA methylation profiles were either publicly available or generated in this study, and for which in most cases, matched gene expression and somatic mutation data were also available (Table 1).

Table 1: Summary of clinicopathological characteristics for five cervical cancer cohorts.

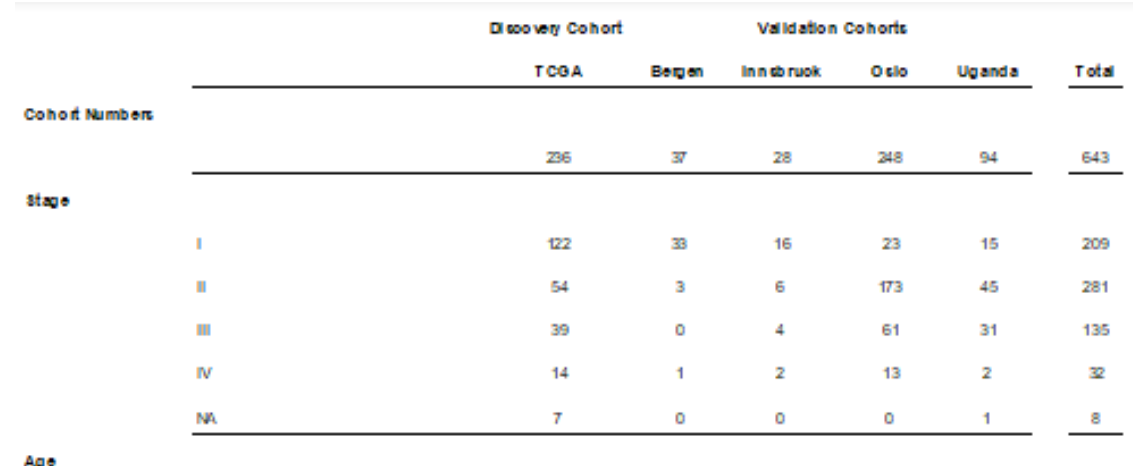

Ago

HPV TYDO

\section{$\operatorname{mosian}(\operatorname{Rang})$}

$47(20 \%)$

$42(2864) \quad 49(2991) \quad 54(2282) \quad 45(2682)$

\begin{tabular}{lllllll}
15 & 136 & 22 & 14 & 158 & 39 & 379 \\
18 & 26 & 2 & 5 & 30 & 14 & $\pi$ \\
45 & 19 & 4 & 0 & 9 & 14 & 45 \\
ouns & 55 & 9 & 9 & 41 & 27 & \\
\hline
\end{tabular}

HPV Clado

\begin{tabular}{|c|c|c|c|c|c|c|}
\hline Aphas 7 & 57 & 6 & 6 & 40 & 32 & 141 \\
\hline Aphas 9 & 172 & 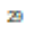 & 18 & 183 & 54 & 456 \\
\hline otora & 7 & 2 & 4 & 25 & 8 & 46 \\
\hline
\end{tabular}

Treetmont

Surgery ano

Surgery and rasidherapy

Surgery, rasolonaty and diemaherspy

Fuscherapy alone

Fosidienapy and dramolnaray

\begin{tabular}{llllll} 
Na & 18 & 5 & 0 & Na & 23 \\
Na & 14 & 17 & 0 & Na & 31 \\
Na & 5 & 6 & 0 & Na & 11 \\
Na & 0 & 0 & 47 & Na & 47 \\
Na & 0 & 0 & 201 & Na & 201 \\
\hline
\end{tabular}

Overall survivel

Mosisn (Finge)

$1.9(0 .-17.5) \quad 8(1.8 .13 .2) \quad 9.8(0.1-23.2) \quad 4.2(0.3 .127) \quad 11(0.2 .4)$

survival statuc

\begin{tabular}{lllllll} 
Alve & 181 & 32 & 16 & 187 & 42 & 458 \\
Deosd & 55 & 5 & 12 & 61 & 52 & \\
\hline
\end{tabular}

Clueter Actignmont

\begin{tabular}{|c|c|c|c|c|c|c|}
\hline $\mathrm{Cl}$ & 175 & 32 & 24 & 198 & 69 & 498 \\
\hline$C 2$ & 61 & 5 & 4 & 50 & 25 & 145 \\
\hline ๙ ${ }^{2} 2$ & 25.8 & 135 & 14.3 & 20.2 & 26.6 & 226 \\
\hline
\end{tabular}

HIV statu c

Avel leb le Dota

\begin{tabular}{llllllll} 
Fog Bve & Na & Na & Na & Na & 59 & \\
Nogatioe & Na & Na & Ma & Na & 35 & & 35 \\
\hline
\end{tabular}

\begin{tabular}{|c|c|c|c|c|c|c|}
\hline RNASEQR & 206 & 3 & Na & Na & 94 & 367 \\
\hline Matiylabon & 206 & 3 & 28 & 248 & 94 & 643 \\
\hline Mration & 206 & 3 & $\mathrm{Na}$ & $\mathrm{Na}$ & 94 & 367 \\
\hline FPA & 137 & $\mathrm{NA}$ & Na & Na & $\mathrm{Na}$ & 137 \\
\hline Moroerray (Alumina HumarWG-6 v3) & $\mathrm{Na}$ & $\mathrm{NA}$ & $\mathrm{Na}$ & 137 & $\mathrm{Na}$ & 137 \\
\hline 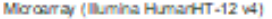 & $\mathrm{NA}$ & $\mathrm{NM}$ & $\mathrm{Na}$ & 109 & $\mathrm{NA}$ & 109 \\
\hline Cogy number (motridaton oriod) & 206 & 3 & 28 & 248 & $\mathrm{Na}$ & 549 \\
\hline
\end{tabular}




\section{Identification of two gene expression-based clusters in cervical squamous cell carcinoma}

Molecular and clinical differences between cervical adeno/adenosquamous and CSCCs are well documented ${ }^{12-14}$ and gene expression differences were apparent in multi-dimensional TSNE analysis based on the top $10 \%$ most variable genes of three previously published cervical cancer cohorts $8,9,11$ with available RNA-seq data (Supplementary Fig. S1a-d). To examine molecular and clinical heterogeneity specifically within SCC we focused all subsequent analysis on a collection of confirmed HPV-positive CSCCs from the USA, Europe and Uganda, as shown in Table 1.

236 cervical SCCs profiled by TCGA were defined as our discovery cohort (Table 1, Supplementary Table S1) and consensus clustering was performed using the top $10 \%$ most variable genes ( $n=1377$ genes, Supplementary Table S2). Consensus cluster membership heatmaps, delta area plot, consensus cumulative distribution function (CDF) and proportion of ambiguous clusters (PAC) indicated the optimal number of clusters was two (Fig. 1a, Supplementary Fig. 2), the larger of which $(n=175)$ was designated C1 while the smaller cluster $(n=61)$ was designated C2 (Supplementary Table S1). Modelling transcriptomic differences between these two clusters identified 938 differentially expressed genes (DEGs, FDR=0.01, FC > 2) (Fig. 1b, Supplementary Table S3). Tumours in $\mathrm{C} 1$ predominantly harbour HPV types from the HPV16-containing alpha-9 clade (150/175) while 38 of 61 C2 tumours contained HPV types from the HPV18-containing alpha-7 clade. C2 tumours were 13.3 times more likely to harbour alpha 7 HPVs than $\mathrm{C} 1$ tumours ( $p=1.8 \times 10^{-14}$, Fishers Exact Test) (Fig. 1b).

Univariate analysis of 5-year overall survival (OS) revealed worse outcomes for patients with C2 tumours $(H R=2.54, p=0.001 ;$ Fig. $1 \mathrm{c})$ and in Cox regression including age, tumour stage and HPV type as covariates along with cluster membership, only membership of the C2 cluster (HR $=2.44, p=0.01795 \%$ $\mathrm{Cl} 1.18,5.05)$ and a tumour stage of IV (HR versus stage I $=4.74, \mathrm{p}<0.001,95 \% \mathrm{Cl} 2.1,10.7)$ were independent predictors of five-year OS (Table 2). The relationship between cluster and OS is also clear when restricting the analysis to HPV16-containing tumours in each cluster in both univariate analysis (HR $=3.39, \mathrm{p}=0.004$; Fig. $1 \mathrm{~d})$ and multivariate analysis, including age and tumour stage as covariates $(\mathrm{HR}=$ $3.89, \mathrm{p}=0.003,95 \% \mathrm{Cl} 1.57,9.67$; Supplementary Table S4).

\section{Identification of C1 and C2 CSCCs and association with prognosis in independent SCC cohorts}

To further investigate the association between C1/C2 cluster membership and OS, we assembled a combined validation cohort consisting of 313 CSCC patients treated at three centres in Europe (Bergen (n = 37), Oslo $(n=248)$ and Innsbruck $(n=28)$ ), for which detailed clinical information were available and for which genome-wide DNA methylation profiles from Illumina Infinium 450k arrays (the same platform used by TCGA) were either available or generated in this study (Table 1). Since RNA-seq data were not available for all European samples, cluster membership was assigned using a support vector machine (SVM) classification model based on $129 \mathrm{CpG}$ sites (methylation variable positions, MVPs) at which methylation differed significantly between tumours in C1 vs C2 clusters in the discovery cohort (Fig. 2a, b; 
mean delta-Beta $>0.25$, FDR $<0.01$, Supplementary Table S5), 18 of which were located within 12 genes differentially expressed between the clusters (Supplementary Fig. S3). MVP and DEG signatures were also used to assign cluster membership to 94 CSCCs from a Ugandan cohort originally profiled by the Cancer Genome Characterization Initiative $(\mathrm{CGCl})^{9}$, for which both DNA methylation and RNA-seq data were available. C2 tumours from all cohorts clustered together using TSNE analysis based on the MVP signature (Fig. 2C) and high concordance between DEG and MVP-based cluster allocation was observed in all cohorts for which both gene expression (RNA-seq for Uganda and Bergen or Illumina bead chip arrays for Oslo) and DNA methylation data were available (Supplementary Fig. S4a, b). Single-sample gene set enrichment analysis (sSGSEA) confirmed differential expression of the signature genes in tumours classified as C1 or C2 using DNA methylation data (Supplementary Fig. 4c). 59 of 313 (18.8\%) tumours in the combined European cohort (Fig. 2b, Supplementary Table S7) and 25 of 94 (26.6\%) tumours in the Ugandan cohort were classified as C2 (Supplementary Fig. S5a, Supplementary Table S6). As in the discovery cohort, most $\mathrm{C} 1$ tumours from the European and Ugandan cohorts harboured alpha-9 HPV types (260/325) while C2 tumours were 3.9 times more likely to harbour alpha-7 HPVs than C1 tumours ( $p=1.07 \times 10^{-6}$, Fishers Exact Test) (Fig. 2b, Supplementary Fig. S5a). Interestingly 80\% (20/25) of Ugandan C2 patients were human immunodeficiency virus (HIV) positive, while only 56\% (39/69) of C1 patients were HIV positive (Supplementary Fig. S5a).

Univariate analysis indicated lower 5-year OS in C2 tumours from the European cohort (Fig. 2d) and Cox regression controlling for FIGO stage, age, HPV type and treatment (surgery alone, surgery with radiochemotherapy, surgery with radiotherapy alone, radio-chemotherapy and radiotherapy alone) again identified C2 status but not HPV type to be an independent predictor of 5-year OS (HR $=2.54, p=0.003$, $95 \% \mathrm{Cl} 1.4,4.7$ ) along with tumour stage and inclusion of chemotherapy in the treatment regimen (Table 2). As in the discovery cohort, a significant prognostic difference was identified between the $\mathrm{C} 1$ and $\mathrm{C} 2$ subgroups when considering only the HPV16-positive tumours $(n=204)$ in both univariate (Supplementary Fig. S5b) and multivariate analyses $(\mathrm{HR}=2.64, \mathrm{p}=0.02,95 \% \mathrm{Cl}=1.16,6$; Supplementary Table S4). Interestingly the prognostic difference was even greater among 78 patients in the European cohort that did not receive chemotherapy (Supplementary Fig. S5c; multivariate HR $=4.4, p=0.005,95 \%$ $\mathrm{Cl}=1.58,12.3)$. At 94 patients, the Ugandan cohort was underpowered for comparing survival between $\mathrm{C} 1$ and $\mathrm{C} 2$ tumours and survival rates in the Ugandan cohort were much lower than in the other cohorts (Supplementary Fig. S5d), thus we did not attempt a combined survival analysis including these patients. Taken together, the C1/C2 clusters identified in the TCGA cohort (USA) are apparent in cohorts of CSCC patients from Europe and Uganda and tumours can be accurately assigned to cluster using either gene expression or DNA methylation profiles. $\mathrm{C} 1 / \mathrm{C} 2$ cluster is an independent predictor of 5-year OS in both the TCGA $(n=236)$ and European $(n=313)$ cohorts and remains so when only HPV16+ tumours are considered. There is no difference in the breakdown of $\mathrm{C} 1$ and $\mathrm{C} 2$ tumours by stage (Supplementary Table S7).

Table 2 - Five-year survival analysis for all cohorts 


\begin{tabular}{|c|c|c|c|c|c|c|}
\hline & \multicolumn{3}{|c|}{ Univariate } & \multicolumn{3}{|c|}{ Multivariate } \\
\hline & Hazard Ratio & p Value & $96 \% \mathrm{Cl}$ & Hazard Ratio & $\mathrm{p}$ Value & $95 \% \mathrm{Cl}$ \\
\hline \multicolumn{7}{|l|}{ TCGA } \\
\hline & 254 & 0.002 & $1.42,4.56$ & 2.44 & 0.02 & $1.18,5.05$ \\
\hline \multicolumn{7}{|l|}{ Bergen } \\
\hline & 5.28 & 0.07 & $0.87,31.9$ & 98.1 & $<0.001$ & $8.41,1145$ \\
\hline \multicolumn{7}{|l|}{ Inn sbruck } \\
\hline & 0 & 1 & $0, \operatorname{lnf}$ & 0 & 1 & $0, \operatorname{Inf}$ \\
\hline \multicolumn{7}{|l|}{ Oslo } \\
\hline & 1.74 & 0.07 & $0.96,3.14$ & 2.36 & 0.012 & $1.21,4.62$ \\
\hline \multicolumn{7}{|l|}{ Eu rope Combined } \\
\hline & 1.68 & 0.07 & $0.97,2.90$ & 2.54 & 0.003 & $1.40,4.67$ \\
\hline \multicolumn{7}{|l|}{ Uganda } \\
\hline & NA & NA & NA & NA & NA & NA \\
\hline
\end{tabular}

\section{Relationships between C1/C2 and clusters previously identified by TCGA}

Of the 178 tumour samples that made up the core set in the TCGA's landmark study into cervical cancer genomics/epigenomics ${ }^{8}, 140$ CSCCs were present in our discovery cohort of 236 (Supplementary Table S8). This enabled comparisons between our gene expression-based cluster allocations and the subtypes defined by TCGA (Fig. 3). TCGA analysis included integrated clustering using multiomics data (three iClusters, two of which ('keratin-high' and 'keratin-low' were composed entirely of CSCCs) and clustering based on transcriptomic data (three mRNA clusters). There is considerable overlap between our $\mathrm{C} 1$ cluster and TCGA's mRNA C2 cluster (84/106) and keratin-high iCluster (80/106), and between our C2 cluster and TCGA's mRNA C3 cluster (19/34) and keratin-low iCluster (27/34). Neither the mRNA C3 nor the keratin-low iCluster were associated with poor prognosis in TCGA's analysis and given the increased expression of a subset of keratin genes (including $K R T 7, K R T 8$ and KRT18) in C2 tumours (Fig. 3), we decided against adopting the keratin-high / keratin-low nomenclature for our clusters. We also examined the relationship between our subtypes and three clusters defined by TCGA based on reverse phase protein array (RPPA) data. Notably, 57\% of C2 TCGA tumours with RPPA data available belong to the EMT cluster compared with only $25 \%$ of $\mathrm{C} 1$ tumours (Fig. 3 ) and, consistent with the proteomic classification, C2 tumours display higher EMT mRNA expression scores, as defined by TCGA ${ }^{8}$ than $\mathrm{C} 1$ tumours (Supplementary Fig. S6). Although there is greater concordance between C2 and the TCGA EMT cluster compared to $\mathrm{C} 1$, it is clearly distinct from the EMT cluster. 


\section{Genomic analyses of prognostic clusters}

To investigate whether $\mathrm{C} 1$ and $\mathrm{C} 2$ tumours differ at the genomic level in addition to the transcriptomic and epigenomic differences observed above, whole-exome data was obtained for SCCs from three cohorts, TCGA $^{8}$, Bergen ${ }^{11}$ and Uganda ${ }^{9}$. This amounted to 367 samples, 29 of which were classed as hypermutated by standards set by TCGA ${ }^{8}(>600$ mutations). The median tumour mutation burden (TMB) was $2.04 / \mathrm{Mb}$ for all tumour, $2.11 / \mathrm{Mb}$ for $\mathrm{C} 1$ tumours and 1.82/Mb for $\mathrm{C} 2$ tumours $(1.92 / \mathrm{Mb}, 1.94 / \mathrm{Mb}$ and $1.72 / \mathrm{Mb}$ respectively after removal of hypermutated samples). We detected four mutation signatures for the combined cohorts (Supplementary Fig. S7): as expected based on previous studies ${ }^{8,9,11}$, COSMIC signatures 2 and 13 (characterised by $C>T$ transitions or $C>G$ transversions respectively at $T p C$ sites attributed to cytosine deamination by APOBEC enzymes); age-related COSMIC signature 1 (characterised by $C>T$ transitions attributed to spontaneous deamination of $5^{\prime}$ methylated cytosine) and COSMIC signature 5 , for which the underlying mutational process is unknown ${ }^{15}$ (https://cancer.sanger.ac.uk/signatures/). The proportion of mutations attributable to each signature did not vary between clusters (Fig. 4).

Having excluded the hypermutated samples, we next performed dNdScv analysis ${ }^{16}$ on each cohort, followed by p-value combination using sample size weighted Fisher's method followed by FDR correction ${ }^{17}$ to permit identification of significantly mutated genes (SMGs) across the entire dataset. This combined approach, followed by analysis of individual samples by cluster identified 34 SMGs (Fig. 4, Supplementary Table S9), 21 of which (highlighted by ${ }^{\dagger}$ ) have not previously been identified as SMGs in cervical cancer $8,9,11$. Of the 34 SMGs, 21 were significantly mutated in only $\mathrm{C} 1$ samples, two genes in only C2 samples, three genes in both $\mathrm{C} 1$ and $\mathrm{C} 2$ individual analysis, and eight genes were only significantly mutated when both $\mathrm{C} 1$ and $\mathrm{C} 2$ clusters were analysed together (Fig. 4, Supplementary Table S9). The frequency of mutations in SMGs that had been previously observed was comparable between combined cohort and each respective SMG study (Supplementary Table S10). Among the 21 genes that have not previously been identified as significantly mutated in cervical cancer, six are SMGs in other SCCs, including head and neck (NOTCH1, JUB (also known as AJUBA), MLL2 (also known as KMT2D), RB1, PIK3R1) ${ }^{18}$, oesophageal (MLL2, NOTCH1, RB1) ${ }^{19}$ and lung SCC (NOTCH1, RB1, MLL2, CREBBP (also known as $K A T 3 A))^{20}$. Conversely, several genes previously identified as SMGs in cervical cancer, including TP53, ARID1A and TGFBR2 are significantly mutated in adenocarcinoma but not in $\mathrm{CSCC}^{8,11}$. Comparing somatic mutation rates in SMGs between clusters using binomial regression identified PIK3CA (FDR $=0.001)$ and EP300 (FDR = 0.046) mutations as disproportionally more common in $\mathrm{C1}$ tumours and STK11 (FDR $=0.005)$ and NF2 (FDR = 0.045) as enriched in C2 tumours (Fig. 4). STK11 is also under-expressed in C2 tumours compared with C1 tumours (Supplementary Table S3).

\section{C2 tumours display Hippo pathway alterations and increased YAP1 activity}

Two SMGs from our analysis (LATS1 and NF2) are core members of the HIPPO signalling pathway, while SMGs FAT1, JUB and STK11 are known regulators of HIPPO signalling ${ }^{21-23}$. Mutations in LATS1, FAT1, 
JUB, STK11 or NF2 (the latter two of which are significantly mutated specifically in C2 tumours, Fig. 4) result in aberrant activation of the downstream transcription factor, yes1 associated transcriptional regulator (YAP1) ${ }^{24-28}$, the expression of which is also elevated at the mRNA level in C2 tumours (Table S3).

We generated segmented copy number data for all tumours (combining TCGA and European validation cohort samples for which the necessary data were available for maximum statistical power), which identified 211 focal candidate copy number alterations (CNAs) at FDR $<0.1$. Following binomial regression, we identified five discrete CNAs that differed in frequency between $\mathrm{C} 1$ and $\mathrm{C} 2$ clusters (Fig. 5a; FDR $<0.1, \log 2$ (Odds Ratio) $>1$ ). All five were more prevalent in $\mathrm{C} 2$ tumours and included 11q11 and 1q21.2 deletions and 6p22.1, 11q22.1 and 11q22.2 gains. 11q22.2 contains matrix metalloproteinase genes (MMPs) which are well known to be involved in metastasis ${ }^{29}$, but notably $11 \mathrm{q} 22.1$ contains the YAP1 gene. Furthermore, analysis of Reverse Phase Protein Assay (RPPA) data from TCGA revealed significantly higher YAP1 protein expression in C2 tumours (Fig. 5b). We confirmed that of the 137 TCGA cases for which RPPA data were available, cases with YAP1 amplification (8/37 C2 tumours and 6/100 $\mathrm{C} 1$ tumours) also showed increased YAP1 mRNA and protein expression (Supplementary Fig. S8). In total 10 genes from a 22 gene signature that predicts HIPPO pathway activity in cancer ${ }^{30}$ are differentially expressed between $\mathrm{C} 1$ and $\mathrm{C} 2$ tumours (Supplementary Table S3).

\section{Differences in the tumour immune microenvironment between $\mathrm{C} 1$ and $\mathrm{C} 2$ tumours.}

The nature of the tumour immune microenvironment, particularly the abundance of tumour infiltrating lymphocytes (TILs) is a strong prognostic factor in cervical cancer ${ }^{31-33}$. We used DNA methylation data to compare the cellular composition of TCGA tumours ${ }^{34}$, observing differences in the proportions of multiple cell types between the subgroups (Fig. 6a); most notably decreased CD8+ (cytotoxic T lymphocytes (CTL)), and a marked elevation of neutrophil and CD56+ natural killer (NK)-cells in C2 tumours. Repeating this method with the validation cohorts produced results that were remarkably similar (Fig. 6b). Differences in the proportions of cell types between $\mathrm{C} 1$ and $\mathrm{C} 2$ in the validation cohort mirrored those in the TCGA cohort, decreased CTL, and elevated neutrophil, NK-cell and endothelial cell levels were observed in C2 tumours. Importantly, this was not driven by any single validation cohort, as individual cohorts displayed consistent patterns of differences in the proportion of cell types between C1 and C2 tumours, especially with regards to CTLs, neutrophils and NK-cells (Supplementary Fig. S9a-d). C2 tumours also exhibit markedly higher neutrophil:CTL ratios (Supplementary Fig. S9e, f) and neutrophil:lymphocyte (CTL, B-cell and Treg) ratios (NLR, Supplementary Fig. S10); established adverse prognostic factors in cervical cancer ${ }^{35-37}$. At 0.7 , the NLR in $\mathrm{C} 1$ tumours across all cohorts was less than half that observed in C2 tumours (1.85).

Validation of MethyICIBERSORT cell estimates was performed for a subset of samples from the Innsbruck cohort using CD8 (CTLs) and myeloperoxidase (MPO, neutrophils) immunohistochemistry (IHC)-based scores from a pathologist blinded to cluster designation (Supplementary Fig. S11a-c) and for 
CTLs in the Oslo cohort samples using comparison of MethyICIBERSORT estimates to CD8 IHC-based digital pathology scores (Supplementary Fig. S11d).

Also of potential significance regarding the tumour immune microenvironment, is the presence of two immune checkpoint genes, $C D 276$ (also known as $B 7-H 3$ ) and NT5E (also known as CD73) in the set of 938 signature DEGs that separate the clusters (Table S3). Both $B 7-H 3$ and NT5E, along with a third immune checkpoint gene ( $P D-L 2)$ are expressed at higher levels in $\mathrm{C} 2$ tumours (Supplementary Fig. S12) and hypomethylation of two CpGs in the NT5E promoter is evident in C2 tumours (Supplementary Table S5). All three suppress T-cell activity ${ }^{38-40}$ and $B 7-H 3$ expression has been linked to poor prognosis in cervical cancer ${ }^{41,42}$.

\section{Evidence for differences in stromal fibroblast phenotype between C1 and C2 tumours}

Gene set enrichment analysis using Metascape ${ }^{43}$ suggested increased EMT (Supplementary Table S9) in C2 tumours, with 52 of 200 genes in in the EMT Hallmark gene set upregulated. As noted above there is also greater overlap between the C2 cluster and an EMT cluster defined by TCGA and based on RPPA data (Figure 3). Single-cell RNA sequencing and xenografting studies strongly suggest that rather than arising from the tumour cells (few of which have undergone EMT at any given time ${ }^{44-46}$ ), mesenchymal gene signatures in bulk tumour expression data instead derive from stromal cells including fibroblasts, which can adopt various phenotypes and play an important role in shaping the tumour immune microenvironment ${ }^{47,48}$. In addition to YAP1, which has been linked to the formation of cancer-associated fibroblasts (CAFs) ${ }^{49}$ (as well as EMT ${ }^{50-52}$ and angiogenesis ${ }^{53}$ ), C2 tumours display increased expression of the CAF marker genes FAP and SERPINE1 (also known as PAI-1) ${ }^{54}$; the latter evidenced at both mRNA and protein levels (Supplementary Table S3, Fig. 5b). Overall fibroblast content as estimated by MethyICIBERSORT is similar between $\mathrm{C} 1$ and $\mathrm{C} 2$ tumours (Fig. 6a, b) but given recent findings regarding the extent and prognostic significance of CAF heterogeneity in the tumour microenvironment ${ }^{55-59}$, we hypothesized that CAF phenotype rather than overall abundance, may differ between $\mathrm{C} 1$ and $\mathrm{C} 2$ tumours. To examine this, hierarchical clustering was performed based on the expression of eight gene sets (68 genes) curated by Qian et $\mathrm{al}^{56}$, representing CAF-related biological processes and which are differentially expressed across six CAF phenotypes recently identified in a pan-cancer analysis ${ }^{59}$. C2 tumours cluster together, displaying increased expression of proinflammatory genes associated with an inflammatory (pan-iCAF2) CAF phenotype, $\mathrm{C} 1$ tumours appear more heterogenous with respect to expression of the signature genes used to define CAF phenotypes; there is upregulation of assorted myofibroblastic (myoCAF) genes in a subgroup $\mathrm{C} 1$ tumours, including various collagens, ECM genes and TGFbassociated genes, as well as 'contractile' genes such as smooth muscle actin (ACTA2, Fig. 6c). While ACTA2 is commonly used to identify myoCAF, it is also expressed by pericytes and smooth muscle cells, which share the contractile phenotype (and express for example, MYH17) ${ }^{47,59,60}$. Consistent with this, C2 tumours are 4.8x ( $p=1.78 \times 10^{-9}$, Fisher's Exact Test) more likely to be classified as 'CAF-high' than C1 tumours using a four-gene CAF index defined by Ko et al ${ }^{48}$. Indeed, three of the four CAF index genes 
(TGFBI, TGFB2 and FN1) appear in the 938 DEG signature that separates C2 from C1 tumours (Supplementary Table S3).

\section{Discussion}

In this study we hypothesized that by drawing upon several cervical cancer cohorts for which 'omics data, clinical information and HPV typing were either available or for which we were able to profile samples ourselves, we would be able to gain further insight into CSCC - the most common histological cervical cancer subtype. Clustering of CSCCs according to the $10 \%$ most variable genes identified two clusters (C1 and $\mathrm{C} 2$ ) that bear resemblance to the keratin-high and keratin-low iClusters originally defined by TCGA ${ }^{8}$. Cluster membership is an independent predictor of 5-year OS and CSCCs can be accurately assigned to cluster using either a 938 gene expression signature or a 129 MVP DNA methylation signature, providing a means by which to gain prognostic information for cervical cancer patients. While HPV16 and the alpha-9 clade to which it belongs have been associated with longer PFS and OS in several studies ${ }^{61-66}$, the relationship between HPV genotype and cervical cancer prognosis remains unclear, as highlighted by a recent meta-analysis ${ }^{7}$. In our multivariate analyses, membership of the $\mathrm{C} 2$ cluster but not HPV type was an independent predictor of poor prognosis in both the discovery and validation cohorts and remained so when only HPV16-positive tumours in either cohort were considered. Possibly, the reason that HPV16 and other alpha-9 HPV types have been associated with more favourable outcomes in certain studies is that these viruses are more likely to cause C1-type tumours.

Adeno- and adenosquamous carcinomas, which are thought to arise from the columnar epithelium of the endocervix, have been linked to poor prognosis in cervical cancer ${ }^{3-6}$ and to avoid differences due to histology, we focused our study entirely on CSCC. Interestingly, of the 14 keratin genes that are differentially expressed between $\mathrm{C} 1$ and $\mathrm{C} 2$ tumours, three (KRT7, KRT8 and KRT18) that are upregulated in $\mathrm{C} 2$ were classified as marker genes for columnar-like tumours with a possible endocervical origin in a recent study that used single cell RNA-sequencing and lineage tracing experiments to explore cell-oforigin for CSCC and adenocarcinoma ${ }^{67}$. In contrast, $\mathrm{C} 1$ tumours display increased expression of $K R T 5$, a marker of the squamous-like subtype with a proposed ectocervical origin identified by Chumduri et al ${ }^{67}$ (Fig. 3). Other signature genes (TP63, CERS3, CSTA, CLCA2, DSC3 and DSG3) upregulated in C1 tumours are also markers of the squamous-like subtype, while further columnar-like marker genes (MUC5B and $R G L 3$ ) are upregulated in $\mathrm{C} 2$ tumours (Supplementary Table S3). Squamous-like tumours are significantly enriched in the $\mathrm{C} 1$ sub-group, a $\mathrm{C} 1$ tumour is $4.9 x$ more likely to be squamous-like than columnar-like or unclassified (Fisher Exact Test, $\mathrm{p}=0.0003$ ). This suggests that $\mathrm{C} 2$ tumours, although SCCs, harbour features associated with adenocarcinoma; possibly even hinting at a different cell-of-origin for $\mathrm{C} 1$ versus $\mathrm{C} 2$ tumours. The greater frequency with which alpha 7 HPV types are found in C2 SCCs is another feature shared with adenocarcinoma.

Our analysis suggests differences in the tumour immune microenvironment between C1 and C2 CSCCs, that are highly reproducible across cohorts from the USA, Europe and Uganda and that might explain the 
differential prognosis associated with these clusters. In addition to the high neutrophil:lymphocyte ratio, the increased expression of cytokines including IL-6, TGF-b and G-CSF and of the chemokines CXCL1-3 in $\mathrm{C} 2$ tumours suggests pro-tumourigenic (N2) polarisation of these neutrophils ${ }^{68-73}$, which is typical of tumours with a high $\mathrm{NLR}^{74}$. The observation that CSCCs occurring in $\mathrm{HIV}^{+}$patients from the Ugandan/CGCl cohort are much more likely to be of the $\mathrm{C} 2$ subtype than those in HIV patients hints at a possible relationship between the immune competence of the patient and the likelihood of developing a $\mathrm{C} 2$ tumour. This requires further investigation but is consistent with greater evidence of existing antitumour immune responses in $\mathrm{C} 1$ tumours.

Finally, it is interesting to note that three targetable immune checkpoint proteins (B7-H3, NT5E and PD-L2) are expressed at higher levels in $\mathrm{C} 2$ tumours. In addition to its immune suppressive effects, $\mathrm{B} 7-\mathrm{H} 3$ has been linked to key processes that are upregulated in these tumours including EMT and angiogenesis, through the activation of NF-kB signalling and the downregulation of E-cadherin expression ${ }^{75,76}$. Interestingly, the expression of B7-H3 and NT5E on CAFs has been linked to poor prognosis in gastric and colorectal cancer, respectively ${ }^{40,77}$. Also of relevance given our observation of differing CAF phenotype between clusters is the report that a CAF subtype (CAF-S1) identified in breast cancer that displays high levels of B7-H3 and NT5E expression is seen in tumours with low levels of CTL infiltration ${ }^{78}$. PD1/PD-L1 immune checkpoint blockade (pembrolizumab) was recently FDA-approved for first-line treatment of metastatic cervical cancer in combination with chemotherapy in patients whose tumours express PDL1 ${ }^{79,80}$, while CTLA4 blockade (Ipilimumab) has also shown promising activity, both as a single agent $^{81,82}$ and in combination with PD1 blockade (Nivolumab) ${ }^{83}$. Efficacy of PD1 blockade in cervical cancer has been linked to the presence of a CD8+FoxP3+CD25+ T-cell subse ${ }^{84}$ and an important limitation of our study is the inability to differentiate between CD8+ T-cell phenotypes. Nonetheless, identification of alternative, targetable immune checkpoint molecules in $\mathrm{C} 2$ tumours provides a potential therapeutic strategy for a subset of cervical cancers that respond poorly to chemoradiotherapy and that, given their low overall levels of T-cell infiltrates, are maybe less likely to respond to PD1 blockade than C1 tumours.

In conclusion, we show that CSCCs can be categorised in two novel tumour types, C1 and C2, among which $\mathrm{C} 1$ tumours have a more favourable outcome. Although HPV16 is more likely to cause $\mathrm{C} 1$ tumours and HPV18 C2 tumours, HPV type is not an independent predictor of prognosis, suggesting it is the tumour type rather than the causative HPV type that is critical for the disease outcome. Notably, the key molecular and cellular characteristics of $\mathrm{C} 1$ and C2 tumours are consistent among cohorts from the US, Europe, and Sub-Saharan Africa. This suggests that the findings and underlying principle: that CSCC can develop along two trajectories associated with differing clinical behaviour that can be identified using defined gene expression or DNA methylation signatures, are of broad relevance.

\section{Methods}

\section{Patient samples}


All patients gave written, informed consent before inclusion. Samples from Bergen were collected in a population-based setting from patients treated at the Department of Obstetrics and Gynaecology, Haukeland University Hospital, Bergen, Norway, from May 2001 to May 2011. The study has been approved by the regional ethical committee (REK 2009/2315, 2014/1907 and 2018/591). For more details on sample collection see11,79. Samples from Innsbruck were collected and processed at the Department of Obstetrics and Gynaecology of the Medical University of Innsbruck. The study was reviewed and approved by the Ethics committee of the Medical University of Innsbruck (reference number: AN2016-0051 360/4.3; 374/5.4: 'Biobank study: Validation of a DNA-methylation based signature in cervical cancer') and conducted in accordance with the Declaration of Helsinki. Samples from Oslo ( $n=$ 268) were collected from patients participating in a previously published prospective clinical study 80 approved by the Regional Committee for Medical Research Ethics in southern Norway (REK no. S-01129). Limited quantities of patient tumour samples and extracted DNA may remain and the distribution of these materials is subject to ethical approval at the institutions from which they were collected. Note that the cases in the Oslo cohort were not treated with surgery. The samples used for molecular analysis were diagnostic biopsies from the primary tumour. In all other cases, specimens were from resections of the primary tumour. Those interested in working with these samples should contact the authors to discuss their requirements.

\section{Dataset assembly}

DNA methylation (Illumina Infinium 450k array) and RNAseq data were obtained for CESC from the TCGA data portal. TCGA mutation data were obtained from the MC3 project on SAGE Synapse (syn7214402). RNAseq data for the Uganda cohort was obtained from the TCGA data portal and DNA methylation (Illumina Infinium EPIC array) and mutation data from National Cancer Institute's Genome Data Commons Publication Page at https://gdc.cancer.gov/about-data/publications/CGCI-HTMCP-CC-2020. DNA methylation (Illumina Infinium 450k array) and gene expression (Illumina HumanHT-12 V4.0 expression beadchip) data from the Oslo cohort were obtained from the Gene Expression Omnibus (GSE68339). RNAseq data were obtained for the Bergen cohort from dbGaP (phs000600/DS-CA-MDS 'Genomic Sequencing of Cervical Cancers') under the authorisation of project \#14589 "Investigating the mechanisms by which viruses and carcinogens contribute to cancer development" and were converted to fastq files using SRA-dump from the SRA Toolkit (http://ncbi.github.io/sra-tools/). Kallisto81 was then used to quantify expression of GENCODE GrCh37 transcripts, repbase repeats and transcripts from 20 different high-risk HPV types with bias correction. Where IDAT files for 450k data were available, they were parsed using minfi82 and were subjected to Functional Normalisation83, followed by BMIQcorrection84 for probe type distribution (which was performed for all methylation data). For TCGA samples, viral type allocation was performed using VirusSeq85.

Only squamous cell carcinomas were considered in this study to avoid confounding from histology. Multidimensional visualisation of the molecular differences in histology was performed using Rtsne $\mathrm{R}$ package with parameters available in Supplementary Table S12, and the top 10\% most variable genes 
using mean absolute deviation after pre filtering of low count genes $(n=1,385)$. Final cohort numbers and summaries are shown in Table 1.

\section{Generation of $450 \mathrm{k}$ methylation profiles}

100ng DNA was bisulphite converted using the EZ DNA Methylation kit (Zymo Research) as per manufacturer's instructions. Bisulphite converted DNA hybridised to the Infinium 450K Human Methylation array and processed in accordance with the manufacturer's recommendations.

\section{HPV typing}

HPV16 or 18 was detected in 208 samples from the Oslo cohort by PCR, using the primers listed in86. The PCR products were detected by polyacrylamide gene electrophoresis or the Agilent DNA 1000 kit (Agilent Technologies Inc, Germany). Samples from the Innsbruck cohort and the remaining nonHPV16/18 samples from the Oslo cohort $(n=40)$ were HPV-typed by DDL Diagnostic Laboratory (Netherlands) using the SPF10 assay, in which a PCR-based detection of over 50 HPV types is followed by a genotyping assay $\left(\right.$ LIPA $\left._{25}\right)$ that identifies 25 HPV types (HPV 6, 11, 16, 18, 31, 33, 34, 35, 39, 40, 42, $43,44,45,51,52,53,54,56,58,59,66,68 / 73,70$ and 74$)$. If more than one HPV type was identified in a sample (e.g. HPV16 and HPV18), that sample was designated "Other" as HPV type in the study. HPV type data for the remaining samples were published previously $8,9,11$.

\section{Prognostic analyses and tumour clustering}

Unsupervised consensus clustering was performed on TCGA SCC samples using r package ConsensusClusterPlus. After prefiltering of genes to remove those with low read counts $(75 \%$ samples read count $<1$ ), only the top $10 \%$ most variable genes using mean absolute deviation were considered for clustering ( $n=1,385)$. 80\% of tumours were sampled over 1000 iterations using all genes. PAM clustering algorithm was used and clustering distance was measured using Pearson's correlation. An optimum number of clusters $(K)$ of 2 was obtained by using the proportion of ambiguously clustered pairs (PAC) using thresholds of 0.1 and 0.9 to define the intermediate sub-interval. PAC was used as it accurately infers K87. Limma-voom on RNAseq data and limma on BMIQ and Functionally-normalised 450k and EPIC data were used to identify differentially expressed genes (DEGs, FDR $=0.01, F C>2$ ) and methylation variable positions (MVPs, FDR $=0.01$, mean delta-Beta $>0.25$ ) between the 2 clusters, $\mathrm{C} 1$ and $\mathrm{C} 2$. The 116 MVPs (Supplementary Table S13) common to the 450k and EPIC arrays were used to allocate clusters for the Ugandan cohort. The mean delta-Beta threshold for MVPs was determined as it delivered the highest concordance between DEG and MVP signature cluster allocation in the Bergen cohort (89.5\%) and high concordance in the Ugandan cohort (91.5\%). The caret R package and limma were used to develop an SVM using 5 iterations of 5 -fold Cross-Validation using DEGs and MVPs to allocate RNAseq samples in Ugandan and Bergen cohorts, 450k samples in Bergen, Innsbruck and Oslo cohorts and EPIC samples in Ugandan cohort to these subgroups. Multidimensional visualisation using R package Rtsne was performed on the TCGA and European cohorts with available DNA methylation data combined using the 129 MVPs and parameters as shown in Supplementary Table S12. 
Samples from our validation cohort, comprise of cases from three European centres (Bergen and Oslo in Norway and Innsbruck, Austria) and one African centre (Uganda) were binned into these categories, and were used for subsequent statistical analyses to identify genomic and microenvironmental correlates. Survival analyses of epigenetic allocations were carried out using Cox Proportional Hazards regression with age, tumour stage, HPV type, and with surgery, radiotherapy and chemotherapy (given/not given) as covariates. R packages used were survival and survminer. For all clinical analyses, stages were collapsed into Stages I, II, III and IV.

RNAseq data for Bergen and Ugandan samples, Illumina HumanWG-6 v3 microarray data for 137 of the Oslo samples and Illumina HumanHT-12 v4 microarray data for 109 of the Oslo samples were used to explore cluster allocation concordance accuracy between DEG and MVP signature cluster allocation. ROC curve and ssGSEA analysis were performed using $\mathrm{R}$ (scripts available at request).

\section{Previous study comparison}

140 TCGA samples from the core set analysis (TCGA, 2017) were present in our TCGA SCC cohort. Previous cluster analysis by TCGA (2017) and Chumduri et al. (2021) was compared with our C1 and C2 cluster allocation.

\section{Pathway analyses}

Pathway and gene sets were analysed with Metascape ${ }^{43}$. Settings used were minimum gene set overlap of $10, p$ value cutoff of 0.01 and minimum enrichment of 1.5. All functional set, pathway, structural complex and miscellaneous gene sets were included in the analysis. Only hits with an FDR of less than 0.05 were included in final results.

\section{Mutational analyses}

For TCGA data, mutation calls were obtained from SAGE synapse as called by the MC3 project. Mutations for the Bergen cohort were obtained from ${ }^{11}$. Ugandan mutation calls were obtained from National Cancer Institute's Genome Data Commons Publication Page at https://gdc.cancer.gov/aboutdata/publications/CGCI-HTMCP-CC-2020. VCFs obtained for the Ugandan cohort samples were converted to maf files using R package vcf2maf, filtered for whole-exome mutations only, and combined. Significantly mutated genes (SMGs) were identified using dNdScv ${ }^{16}$ individually for the three cohorts. Hypermutated samples $\left(>600\right.$ mutations $\left.^{8}\right)$ were excluded from this analysis. A weighted approach was used to combine $p$ values for each gene for the three cohorts. R package metapro ${ }^{17}$ function wFisher was used to perform this task. Genes were considered SMGs if after FDR correction of combined $\mathrm{p}$ values, $\mathrm{q}<$ 0.1. Analysis was repeated for only $\mathrm{C} 1$ and $\mathrm{C} 2$ samples individually. Two genes were removed from our list. MUC4 was removed due to the large size of the gene and GOLGA6L 18 was removed as this gene and it's aliases were not recognised by R package maftools 88 . 
R package maftools was used to produce an oncoplot for SMGs, calculating tumour mutational burden for individual samples, SMG mutation frequency and mutational signatures for the combined cohorts. Binomial GLMs were used to estimate associations between C1 and C2 clusters and SMG mutation frequencies.

The estimated exposures of each sample to the identified mutational signatures were calculated using $\mathrm{R}$ package mutsignatures 89 and converted to proportion of signature exposure per sample.

\section{Copy number analysis}

450k total intensities (Methylated and Unmethylated values) were used to generate copy number profiles with normal blood samples from Renius et al90 as the germline reference. Functional normalisation83 was used to regress out technical variation across the reference and tumour datasets before merging and quantile normalisation was used to normalise combined intensities followed by Circular Binary Segmentation as previously described 91 . Median density peak correction was performed to ensure centering before further analysis. GISTIC2.092 was then used to identify regions of significant copy number change at both arm and gene levels. Candidate copy number changes were evaluated for association with cluster using binomial GLMs. The parameters chosen were a noise threshold of 0.1 with arm-level peel off and a confidence level of 0.95 was used to nominate genes targeted by copy number changes. Binomial regression was finally used to estimate rates of differential alteration.

\section{Reverse Phase Protein Assay analysis}

Reverse Phase Protein Assay (RPPA) data for the core TCGA CESC samples were obtained from the NCl GDC Legacy Archive. Differentially expressed proteins between $\mathrm{C} 1$ and $\mathrm{C} 2$ clusters were determined using R package limma (FDR $=0.05, F C>1.3)$.

\section{Tumour microenvironment analyses}

MethyICIBERSORT ${ }^{34}$ was used to estimate tumour purity and abundances of nine other microenvironmental cellular fractions using TCGA and validation cohort methylation beta values. Fraction numbers were then normalised by cellular abundance and differences between clusters $\mathrm{C} 1$ and C2 were estimated using Wilcoxon's rank sum test with Benjamini Hochberg correction for multiple testing. This analysis was performed separately on TCGA cohort and combined validation cohort, as well as on each individual cohort.

Cancer associated fibroblast associated gene set lists were obtained from Qian et al ${ }^{56}$. TCGA, Bergen and Ugandan cohort sample RNAseq data was combined and visualised for these gene set genes using $\mathrm{R}$ package NMF93.

\section{CAF Index calculation}


For cohorts that RNAseq data was available (TCGA, Bergen and Uganda), a CAF index was calculated as described in $\mathrm{Ko}$ et $\mathrm{al}^{48}$. The median $\mathrm{CAF}$ index value was used as a threshold to allocate high or low CAF in tumour samples.

\section{Immunohistochemistry}

Immunohistochemical staining of samples from the Innsbruck cohort was conducted by HSL-Advanced Diagnostics (London, UK) using the Leica Bond III platform with Leica Bond Polymer Refine detection as per manufacturer's recommendations. Sections from a series of 17 tumour samples from the validation cohort were stained for CD8 (mouse monoclonal 4B11, Leica Biosystems PA0183, used as supplied for 15 minutes at room temperature. HIER was performed on-board using Leica ER2 solution (high pH) for 20 minutes), CD68 (mouse monoclonal PGM1, Agilent M087601-2, used at a dilution of 1/50 for 15mins at room temperature. HIER was performed on-board using Leica ER1 solution (low pH) for 20 minutes) or MPO (rabbit polyclonal, Agilent A039829-2, used at a dilution of 1/4000 for 15 minutes at room temperature without epitope retrieval. Scoring was performed blinded to cluster membership by a histopathologist $(\mathrm{JM})$ as follows: $0=$ no positive cells $/$ field (200X magnification); $1=1-10$ positive cells; $2=11-100$ positive cells; $3=101-200$ positive cells; $4=201=300$ positive cells; $5=$ over 300 positive cells.

For the Oslo cohort, manual CD8 staining was conducted using the Dako EnVision ${ }^{\mathrm{TM}}$ Flex+ System (K8012, Dako). Deparaffinization and unmasking of epitopes were performed using PT-Link (Dako) and EnVision ${ }^{\mathrm{TM}}$ Flex target retrieval solution at a high $\mathrm{pH}$. The sections were incubated with CD8 mouse monoclonal antibody (clone 4B11, 1:150, $0.2 \mu \mathrm{g} \mathrm{IgG}_{2 \mathrm{~b}} / \mathrm{ml}$ ) from Novocastra (Leica Microsystems, Newcastle Upon Tyne, UK) for 45 minutes. All CD8 series included positive controls. Negative controls included substitution of the monoclonal antibody with mouse myeloma protein of the same subclass and concentration as the monoclonal antibody. All controls gave satisfactory results. CD8 pathology scores were given to each sample (blinded to cluster membership) for connective tissue only, tumour only and both as follows: $0=$ no positive: $1=<10 \%$ CD8 positive cells; $2=10-25 \%$ CD 8 positive cells; $3=25-50 \%$ CD8 positive cells; $4=>50 \%$ CD 8 positive cells. For digital quantification scanned images of all sections at a high resolution of $0.46 \mathrm{um} /$ pixel (20x), which was reduced to $0.92 \mathrm{um} /$ pixel for analysis, were used. Digital score was calculated by quantifying the area fraction of stained CD8 cells in relation to the entire section in the digital assessment.

\section{Declarations}

\section{Data availability}

Illumina Infinium 450k array DNA methylation data generated in-house from Bergen and Innsbruck validation cohort samples have been deposited in the Gene Expression Omnibus (accession number GSEXXXXX (to be deposited upon publication)). For detailed information on all other datasets see 'Dataset Assembly'. 


\section{Code availability}

All packages used have been published, are freely available and are referenced in the methods. $R$ markdowns used to run the analyses specific to this study are available from the authors on request.

\section{Acknowledgements}

AC was supported by postgraduate research scholarships from UCL and received additional research support from a Debbie Fund grant to KC and TRF. TRF was supported by Rosetrees Trust (M229-CD1), Cancer Research UK (A25825), the Biotechnology and Biosciences Research Council (Grant Ref: BB/V010271/1), the Royal Society (IEC\R2\202256) and the Global Challenges Doctoral Centre at the University of Kent. DNA methylation data were generated through funding provided by the Debbie Fund and the results shown here are in part based upon data generated by the TCGA Research Network: https://www.cancer.gov/tcga and the Cancer Genome Characterization Initiative: https://ocg.cancer.gov/programs/cgci. AF was supported by grants from the MRC (MR/M025411/1), PCUK(MA-TR15-009), BBSRC (BB/R009295/1), TUF, Orchid and the UCLH BRC. The authors dedicate this manuscript to the late Dr Helga Salvesen, a wonderful collaborator and colleague who played a key role in the project.

\section{References}

1. de Martel, C., Plummer, M., Vignat, J. \& Franceschi, S. Worldwide burden of cancer attributable to HPV by site, country and HPV type. International journal of cancer 141, 664-670 (2017).

2. Li, N., Franceschi, S., Howell-Jones, R., Snijders, P. J. F. \& Clifford, G. M. Human papillomavirus type distribution in 30,848 invasive cervical cancers worldwide: Variation by geographical region, histological type and year of publication. International journal of cancer 128, 927-935 (2011).

3. Jung, E. J. et al. Cervical adenocarcinoma has a poorer prognosis and a higher propensity for distant recurrence than squamous cell carcinoma. International Journal of Gynecological Cancer 27, 12281236 (2017).

4. Huang, Y. T. et al. Clinical behaviors and outcomes for adenocarcinoma or adenosquamous carcinoma of cervix treated by radical hysterectomy and adjuvant radiotherapy or chemoradiotherapy. International Journal of Radiation Oncology Biology Physics 84, 420-427 (2012).

5. Zhou, J. et al. Comparison of clinical outcomes of squamous cell carcinoma, adenocarcinoma, and adenosquamous carcinoma of the uterine cervix after definitive radiotherapy: a population-based analysis. Journal of cancer research and clinical oncology 143, 115-122 (2017).

6. Galic, V. et al. Prognostic significance of adenocarcinoma histology in women with cervical cancer. Gynecologic Oncology 125, 287-291 (2012).

7. Chen, X. et al. Better or Worse? The Independent Prognostic Role of HPV-16 or HPV-18 Positivity in Patients With Cervical Cancer: A Meta-Analysis and Systematic Review. Frontiers in oncology vol. 10 
1733 (2020).

8. Burk, R. D. et al. Integrated genomic and molecular characterization of cervical cancer. Nature 543, 378-384 (2017).

9. Gagliardi, A. et al. Analysis of Ugandan cervical carcinomas identifies human papillomavirus cladespecific epigenome and transcriptome landscapes. Nature genetics 52, 800-810 (2020).

10. Huang, J. et al. Comprehensive genomic variation profiling of cervical intraepithelial neoplasia and cervical cancer identifies potential targets for cervical cancer early warning. Journal of medical genetics 56, 186-194 (2019).

11. Ojesina, A. I. et al. Landscape of genomic alterations in cervical carcinomas. Nature 506, 371-375 (2014).

12. Chen, J. L. et al. Differential clinical characteristics, treatment response and prognosis of locally advanced adenocarcinoma/adenosquamous carcinoma and squamous cell carcinoma of cervix treated with definitive radiotherapy. Acta Obstetricia et Gynecologica Scandinavica 93, 661-668 (2014).

13. Williams, N. L., Werner, T. L., Jarboe, E. A. \& Gaffney, D. K. Adenocarcinoma of the cervix: should we treat it differently? Current oncology reports 17, 16-17 (2015).

14. Hu, K., Wang, W., Liu, X., Meng, Q. \& Zhang, F. Comparison of treatment outcomes between squamous cell carcinoma and adenocarcinoma of cervix after definitive radiotherapy or concurrent chemoradiotherapy. Radiation oncology (London, England) 13, 245-249 (2018).

15. Alexandrov, L. B. et al. Signatures of mutational processes in human cancer. Nature 500, 415-421 (2013).

16. Martincorena, I. et al. Universal Patterns of Selection in Cancer and Somatic Tissues. Cell 171, 10291041.e21 (2017).

17. Yoon, S., Baik, B., Park, T. \& Nam, D. Powerful p-value combination methods to detect incomplete association. Scientific Reports 11, 6980 (2021).

18. Lawrence, M. S. et al. Comprehensive genomic characterization of head and neck squamous cell carcinomas. Nature 517, 576-582 (2015).

19. Lin, D.-C. et al. Genomic and molecular characterization of esophageal squamous cell carcinoma. Nature genetics 46, 467-473 (2014).

20. Hammerman, P. S. et al. Comprehensive genomic characterization of squamous cell lung cancers. Nature 489, 519-525 (2012).

21. Rauskolb, C., Sun, S., Sun, G., Pan, Y. \& Irvine, K. D. Cytoskeletal tension inhibits Hippo signaling through an Ajuba-Warts complex. Cell 158, 143-156 (2014).

22. Nguyen, T. H., Ralbovska, A. \& Kugler, J.-M. RhoBTB Proteins Regulate the Hippo Pathway by Antagonizing Ubiquitination of LKB1. G3 (Bethesda, Md.) 10, 1319-1325 (2020).

23. Mohseni, M. et al. A genetic screen identifies an LKB1-MARK signalling axis controlling the HippoYAP pathway. Nature cell biology 16, 108-117 (2014). 
24. Martin, D. et al. Assembly and activation of the Hippo signalome by FAT1 tumor suppressor. Nature communications 9, 2372 (2018).

25. Sourbier, C. et al. Targeting loss of the Hippo signaling pathway in NF2-deficient papillary kidney cancers. Oncotarget 9, 10723-10733 (2018).

26. Petrilli, A. M. \& Fernández-Valle, C. Role of Merlin/NF2 inactivation in tumor biology. Oncogene 35, 537-548 (2016).

27. White, S. M. et al. YAP/TAZ Inhibition Induces Metabolic and Signaling Rewiring Resulting in Targetable Vulnerabilities in NF2-Deficient Tumor Cells. Developmental cell 49, 425-443.e9 (2019).

28. Yang, H. et al. NF2 and Canonical Hippo-YAP Pathway Define Distinct Tumor Subsets Characterized by Different Immune Deficiency and Treatment Implications in Human Pleural Mesothelioma. Cancers 13, 1561. doi: 10.3390/cancers13071561 (2021).

29. Gonzalez-Avila, G. et al. Matrix metalloproteinases participation in the metastatic process and their diagnostic and therapeutic applications in cancer. Critical reviews in oncology/hematology 137, 5783 (2019).

30. Wang, Y. et al. Comprehensive Molecular Characterization of the Hippo Signaling Pathway in Cancer. Cell reports 25, 1304-1317.e5 (2018).

31. Gooden, M. J., de Bock, G. H., Leffers, N., Daemen, T. \& Nijman, H. W. The prognostic influence of tumour-infiltrating lymphocytes in cancer: a systematic review with meta-analysis. British journal of cancer 105, 93-103 (2011).

32. Jordanova, E. S. et al. Human leukocyte antigen class I, MHC class I chain-related molecule A, and $\mathrm{CD} 8+/$ regulatory T-cell ratio: which variable determines survival of cervical cancer patients? Clinical cancer research: an official journal of the American Association for Cancer Research 14, 2028-2035 (2008).

33. Nedergaard, B. S., Ladekarl, M., Thomsen, H. F., Nyengaard, J. R. \& Nielsen, K. Low density of CD3+, $\mathrm{CD} 4+$ and $\mathrm{CD} 8+$ cells is associated with increased risk of relapse in squamous cell cervical cancer. British journal of cancer 97, 1135-1138 (2007).

34. Chakravarthy, A. et al. Pan-cancer deconvolution of tumour composition using DNA methylation. Nature communications 9, 3220-3221 (2018).

35. Mizunuma, M. et al. The pretreatment neutrophil-to-lymphocyte ratio predicts therapeutic response to radiation therapy and concurrent chemoradiation therapy in uterine cervical cancer. International journal of clinical oncology 20, 989-996 (2015).

36. Lee, Y. Y. et al. Pretreatment neutrophil:lymphocyte ratio as a prognostic factor in cervical carcinoma. Anticancer Research 32, 1555-1561 (2012).

37. Huang, Q. T. et al. Prognostic significance of neutrophil-to-lymphocyte ratio in cervical cancer: A systematic review and meta-analysis of observational studies. Oncotarget 8, 16755-16764 (2017).

38. Prasad, D. V. R. et al. Murine B7-H3 Is a Negative Regulator of T Cells. The Journal of Immunology 173,2500 (2004). 
39. Zang, X. et al. B7x: a widely expressed B7 family member that inhibits $\mathrm{T}$ cell activation. Proceedings of the National Academy of Sciences of the United States of America 100, 10388-10392 (2003).

40. Yu, M. et al. CD73 on cancer-associated fibroblasts enhanced by the A2B-mediated feedforward circuit enforces an immune checkpoint. Nature Communications 11, 515 (2020).

41. Han, S. et al. Roles of B7-H3 in Cervical Cancer and Its Prognostic Value. Journal of Cancer 9, 26122624 (2018).

42. Huang, C. et al. B7-H3, B7-H4, Foxp3 and IL-2 expression in cervical cancer: Associations with patient outcome and clinical significance. Oncology reports 35, 2183-2190 (2016).

43. Zhou, Y. et al. Metascape provides a biologist-oriented resource for the analysis of systems-level datasets. Nature communications 10, 1523-1526 (2019).

44. Ruscetti, M., Quach, B., Dadashian, E. L., Mulholland, D. J. \& Wu, H. Tracking and Functional Characterization of Epithelial-Mesenchymal Transition and Mesenchymal Tumor Cells during Prostate Cancer Metastasis. Cancer research 75, 2749-2759 (2015).

45. Fischer, K. R. et al. Epithelial-to-mesenchymal transition is not required for lung metastasis but contributes to chemoresistance. Nature 527, 472-476 (2015).

46. Zheng, X. et al. Epithelial-to-mesenchymal transition is dispensable for metastasis but induces chemoresistance in pancreatic cancer. Nature 527, 525-530 (2015).

47. Puram, S. v et al. Single-Cell Transcriptomic Analysis of Primary and Metastatic Tumor Ecosystems in Head and Neck Cancer. Cell 171, 1611-1624.e24 (2017).

48. Ko, Y.-C. et al. Index of Cancer-Associated Fibroblasts Is Superior to the Epithelial-Mesenchymal Transition Score in Prognosis Prediction. Cancers 12, 1718 (2020).

49. Shen, T. et al. YAP1 plays a key role of the conversion of normal fibroblasts into cancer-associated fibroblasts that contribute to prostate cancer progression. Journal of Experimental \& Clinical Cancer Research 39, 36 (2020).

50. Zanconato, F. et al. Genome-wide association between YAP/TAZ/TEAD and AP-1 at enhancers drives oncogenic growth. Nature cell biology 17, 1218-1227 (2015).

51. Shao, D. D. et al. KRAS and YAP1 converge to regulate EMT and tumor survival. Cell 158, 171-184 (2014).

52. Schlegelmilch, K. et al. Yap1 acts downstream of a-catenin to control epidermal proliferation. Cell 144, 782-795 (2011).

53. Kim, J. et al. YAP/TAZ regulates sprouting angiogenesis and vascular barrier maturation. The Journal of clinical investigation 127, 3441-3461 (2017).

54. Sakamoto, H. et al. PAl-1 derived from cancer-associated fibroblasts in esophageal squamous cell carcinoma promotes the invasion of cancer cells and the migration of macrophages. Laboratory Investigation 101, 353-368 (2021).

55. Neuzillet, C. et al. Inter- and intra-tumoural heterogeneity in cancer-associated fibroblasts of human pancreatic ductal adenocarcinoma. The Journal of pathology 248, 51-65 (2019). 
56. Qian, J. et al. A pan-cancer blueprint of the heterogeneous tumor microenvironment revealed by single-cell profiling. Cell research 30, 745-762 (2020).

57. Mhaidly, R. \& Mechta-Grigoriou, F. Fibroblast heterogeneity in tumor micro-environment: Role in immunosuppression and new therapies. Seminars in Immunology 48, 101417 (2020).

58. Hutton, C. et al. Single-cell analysis defines a pancreatic fibroblast lineage that supports anti-tumor immunity. Cancer cell 39, 1227-1244.e20 (2021).

59. Galbo, P. M., Zang, X. \& Zheng, D. Molecular Features of Cancer-associated Fibroblast Subtypes and their Implication on Cancer Pathogenesis, Prognosis, and Immunotherapy Resistance. Clinical Cancer Research 27, 2636 (2021).

60. Chen, Z. et al. Single-cell RNA sequencing highlights the role of inflammatory cancer-associated fibroblasts in bladder urothelial carcinoma. Nature Communications 11, 5077 (2020).

61. Rader, J. S. et al. Genetic variations in human papillomavirus and cervical cancer outcomes. International journal of cancer 144, 2206-2214 (2019).

62. Wright, J. D. et al. Human papillomavirus type and tobacco use as predictors of survival in early stage cervical carcinoma. Gynecologic oncology 98, 84-91 (2005).

63. Yang, S. H., Kong, S. K., Lee, S. H., Lim, S. Y. \& Park, C. Y. Human papillomavirus 18 as a poor prognostic factor in stage I-IIA cervical cancer following primary surgical treatment. Obstetrics \& gynecology science $57,492-500$ (2014).

64. Burger, R. A. et al. Human papillomavirus type 18: association with poor prognosis in early stage cervical cancer. Journal of the National Cancer Institute 88, 1361-1368 (1996).

65. Schwartz, S. M. et al. Human papillomavirus and prognosis of invasive cervical cancer: a populationbased study. Journal of clinical oncology: official journal of the American Society of Clinical Oncology 19, 1906-1915 (2001).

66. Hang, D. et al. Independent prognostic role of human papillomavirus genotype in cervical cancer. BMC Infectious Diseases 17, 391 (2017).

67. Chumduri, C. et al. Opposing Wnt signals regulate cervical squamocolumnar homeostasis and emergence of metaplasia. Nature cell biology 23, 184-197 (2021).

68. Fridlender, Z. G. et al. Polarization of tumor-associated neutrophil phenotype by TGF-beta: "N1" versus “N2" TAN. Cancer cell 16, 183-194 (2009).

69. Zhu, Q. et al. The IL-6-STAT3 axis mediates a reciprocal crosstalk between cancer-derived mesenchymal stem cells and neutrophils to synergistically prompt gastric cancer progression. Cell Death \& Disease 5, e1295-e1295 (2014).

70. Ohms, M., Möller, S. \& Laskay, T. An Attempt to Polarize Human Neutrophils Toward N1 and N2 Phenotypes in vitro. Frontiers in immunology 11, 532 (2020).

71. SenGupta, S. et al. Triple-Negative Breast Cancer Cells Recruit Neutrophils by Secreting TGF- $\beta$ and CXCR2 Ligands. Frontiers in immunology 12, 659996 (2021). 
72. Casbon, A. J. et al. Invasive breast cancer reprograms early myeloid differentiation in the bone marrow to generate immunosuppressive neutrophils. Proceedings of the National Academy of Sciences of the United States of America 112, E566-75 (2015).

73. Shaul, M. E. et al. Tumor-associated neutrophils display a distinct N1 profile following TGF $\beta$ modulation: A transcriptomics analysis of pro- vs. antitumor TANs. Oncoimmunology 5, e1232221 (2016).

74. Kim, Y., Lee, D., Lee, J., Lee, S. \& Lawler, S. Role of tumor-associated neutrophils in regulation of tumor growth in lung cancer development: A mathematical model. PloS one 14, e0211041 (2019).

75. Xie, C. et al. Soluble B7-H3 promotes the invasion and metastasis of pancreatic carcinoma cells through the TLR4/NF-kB pathway. Scientific reports 6, 27528 (2016).

76. MacGregor, H. L. et al. High expression of B7-H3 on stromal cells defines tumor and stromal compartments in epithelial ovarian cancer and is associated with limited immune activation. Journal for ImmunoTherapy of Cancer 7, 357 (2019).

77. Zhan, S. et al. Overexpression of B7-H3 in a-SMA-Positive Fibroblasts Is Associated With Cancer Progression and Survival in Gastric Adenocarcinomas. Frontiers in Oncology 9, 1466 (2020).

78. Costa, A. et al. Fibroblast Heterogeneity and Immunosuppressive Environment in Human Breast Cancer. Cancer cell 33, 463-479.e10 (2018).

79. Chung, H. C. et al. Efficacy and Safety of Pembrolizumab in Previously Treated Advanced Cervical Cancer: Results From the Phase II KEYNOTE-158 Study. Journal of Clinical Oncology 37, 1470-1478 (2019).

80. Colombo, N. et al. Pembrolizumab for Persistent, Recurrent, or Metastatic Cervical Cancer. New England Journal of Medicine (2021) doi:10.1056/nejmoa2112435.

81. Lheureux, S. et al. Association of Ipilimumab With Safety and Antitumor Activity in Women With Metastatic or Recurrent Human Papillomavirus-Related Cervical Carcinoma. JAMA oncology 4, e173776-e173776 (2018).

82. Mayadev, J. S. et al. Sequential Ipilimumab After Chemoradiotherapy in Curative-Intent Treatment of Patients With Node-Positive Cervical Cancer. JAMA oncology 6, 92-99 (2020).

83. Naumann, R. W. et al. Efficacy and safety of nivolumab (Nivo) + ipilimumab (Ipi) in patients (pts) with recurrent/metastatic (R/M) cervical cancer: Results from CheckMate 358. in Annals of Oncology v898-v899 (2019). doi:10.1093/annonc/mdz394.

84. Heeren, A. M. et al. Efficacy of PD-1 blockade in cervical cancer is related to a CD8+FoxP3+CD25+ Tcell subset with operational effector functions despite high immune checkpoint levels. Journal for ImmunoTherapy of Cancer 7, 43 (2019).

85. Halle, M. K. et al. Clinicopathologic and molecular markers in cervical carcinoma: a prospective cohort study. American journal of obstetrics and gynecology 217, 432.e1-432.e17 (2017).

86. Lando, M. et al. Identification of eight candidate target genes of the recurrent 3p12-p14 loss in cervical cancer by integrative genomic profiling. The Journal of pathology 230, 59-69 (2013). 
87. Bray, N. L., Pimentel, H., Melsted, P. \& Pachter, L. Near-optimal probabilistic RNA-seq quantification. Nature Biotechnology 34, 525-527 (2016).

88. Aryee, M. J. et al. Minfi: a flexible and comprehensive Bioconductor package for the analysis of Infinium DNA methylation microarrays. Bioinformatics (Oxford, England) 30, 1363-1369 (2014).

89. Fortin, J.-P. et al. Functional normalization of $450 \mathrm{k}$ methylation array data improves replication in large cancer studies. Genome biology 15, 503 (2014).

90. Teschendorff, A. E. et al. A beta-mixture quantile normalization method for correcting probe design bias in Illumina Infinium 450 k DNA methylation data. Bioinformatics (Oxford, England) 29, 189-196 (2013).

91. Chen, Y. et al. VirusSeq: software to identify viruses and their integration sites using next-generation sequencing of human cancer tissue. Bioinformatics (Oxford, England) 29, 266-267 (2013).

92. Lyng, H. et al. Intratumor chromosomal heterogeneity in advanced carcinomas of the uterine cervix. International Journal of Cancer 111, 358-366 (2004).

93. Șenbabaoğlu, Y., Michailidis, G. \& Li, J. Z. Critical limitations of consensus clustering in class discovery. Scientific reports 4, 6207 (2014).

94. Mayakonda, A., Lin, D.-C., Assenov, Y., Plass, C. \& Koeffler, H. P. Maftools: efficient and comprehensive analysis of somatic variants in cancer. Genome research 28, 1747-1756 (2018).

95. Fantini, D., Vidimar, V., Yu, Y., Condello, S. \& Meeks, J. J. MutSignatures: an R package for extraction and analysis of cancer mutational signatures. Scientific Reports 10, 18217 (2020).

96. Reinius, L. E. et al. Differential DNA methylation in purified human blood cells: implications for cell lineage and studies on disease susceptibility. PloS one 7, e41361-e41361 (2012).

97. Feber, A. et al. Using high-density DNA methylation arrays to profile copy number alterations. Genome biology 15, R30-R30 (2014).

98. Mermel, C. H. et al. GISTIC2.0 facilitates sensitive and confident localization of the targets of focal somatic copy-number alteration in human cancers. Genome Biology 12, R41 (2011).

99. Gaujoux, R. \& Seoighe, C. A flexible R package for nonnegative matrix factorization. BMC Bioinformatics 11, 367 (2010).

\section{Supplementary Tables}

Table S1 - Clinical and pathaologic characteristics of TCGA squamous cervical cancer cohort samples

Table S2 - Top 10\% most variable genes in TCGA squamous cervical cancer cohort

Table S3 - 938 Differentially expressed genes between TCGA squamous cervical cancer clusters C1 and c2

Table S4 - 5 year survival uni- and multivariate analysis for HPV16+ patients in squamous cervical cancer cohorts 
Table S5 - 129 MVP signature probes (European validation cohorts)

Table S6 - Combined validation cohort cluster allocation

Table S7 - Breakdown of tumour stage in C1 and C2 cluster by percentage

Table S8 - Clusters and EMT scores for TCGA squamous cervical cancer samples

Table S9 - Significantly mutated genes using dNdSCV analysis and combining cohorts

Table S10 - Mutation frequency in SMGs observed in previous studies

Table 11 - Gene set enrichment analysis of C2 gene expression signature genes using Metascape

Table S12 - Paramaters for TSNE multidimensional visualisation analyses

Table S13 - 116 MVP signature probes (Ugandan validation cohort)

\section{Figures}


a

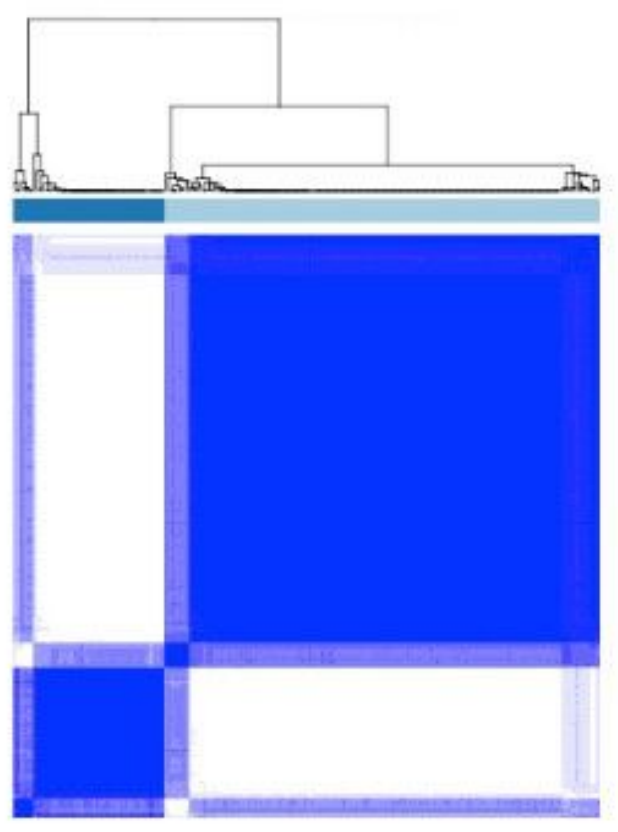

C
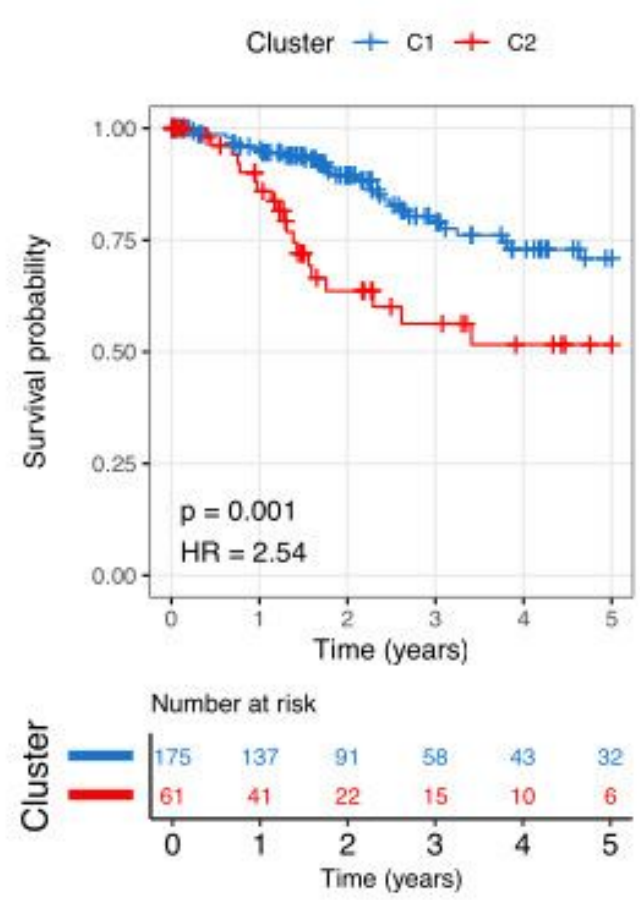

b
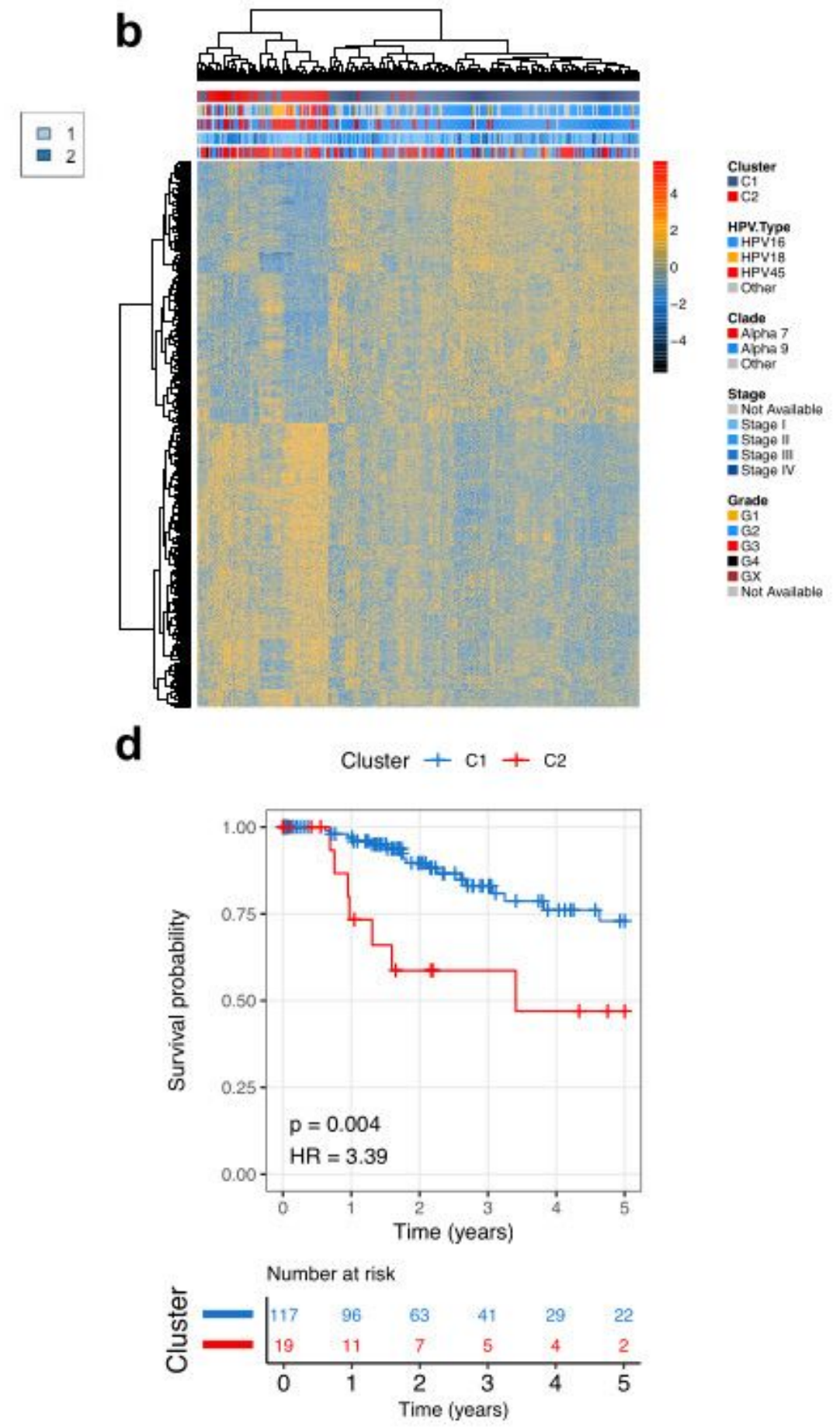

Figure 1 Consensus clustering produces two prognostic clusters in TCGA SCC cohort. a) Consensus clustering of 236 TCGA HPV+ SCC patients. b) There were 938 differentially expressed genes between the two clusters. c) 5 year survival between the 2 SCC subgroups. d) 5 year survival between the 2 SCC subgroups considering only HPV16+ tumours. Statistics from univariate Cox regression.

\section{Figure 1}

See image above for figure legend. 


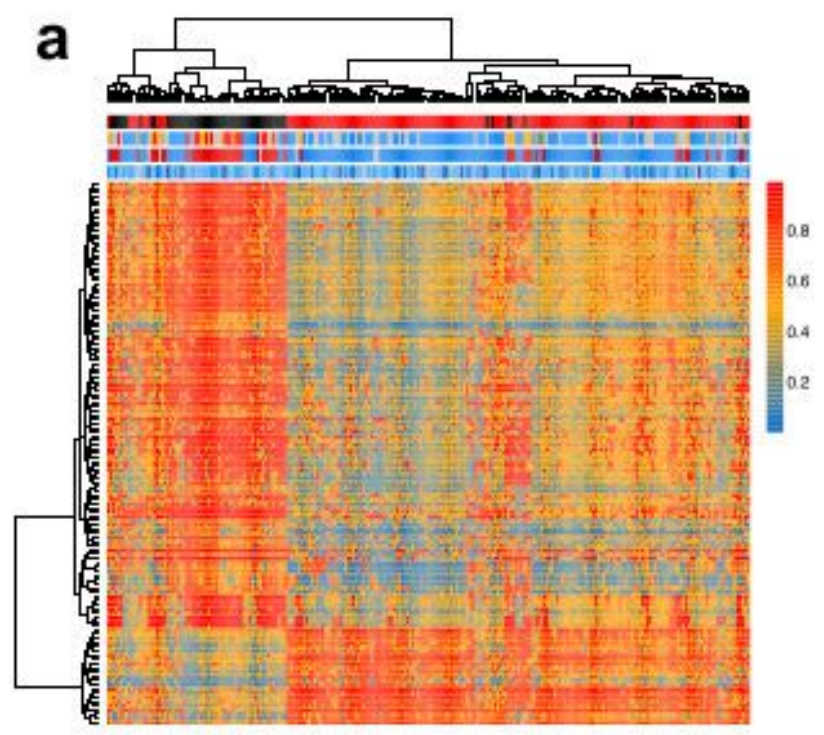

C

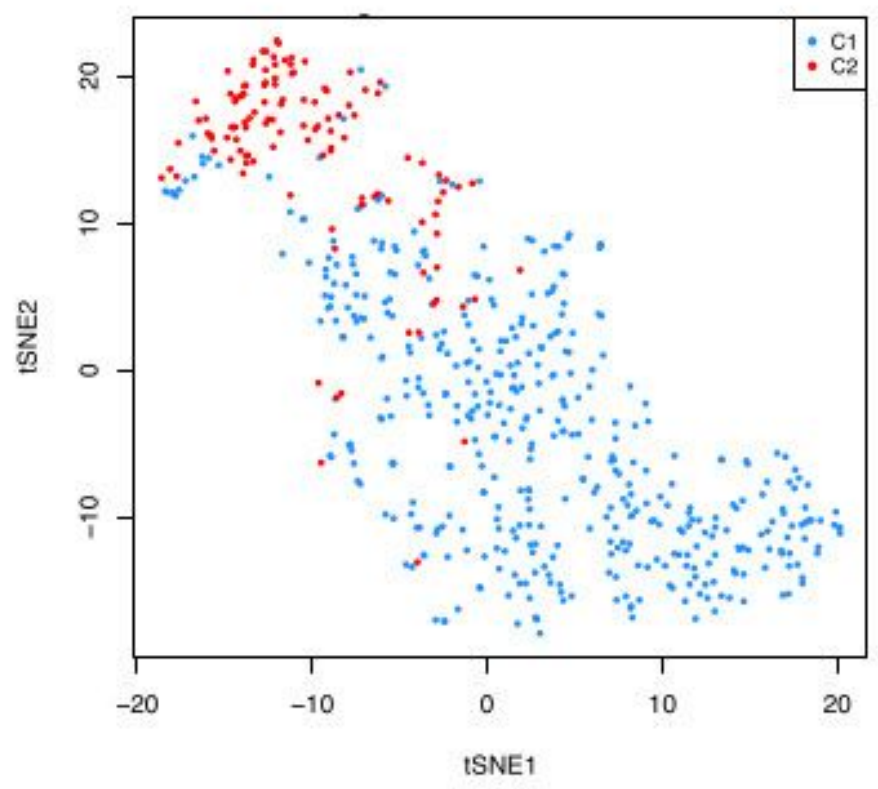

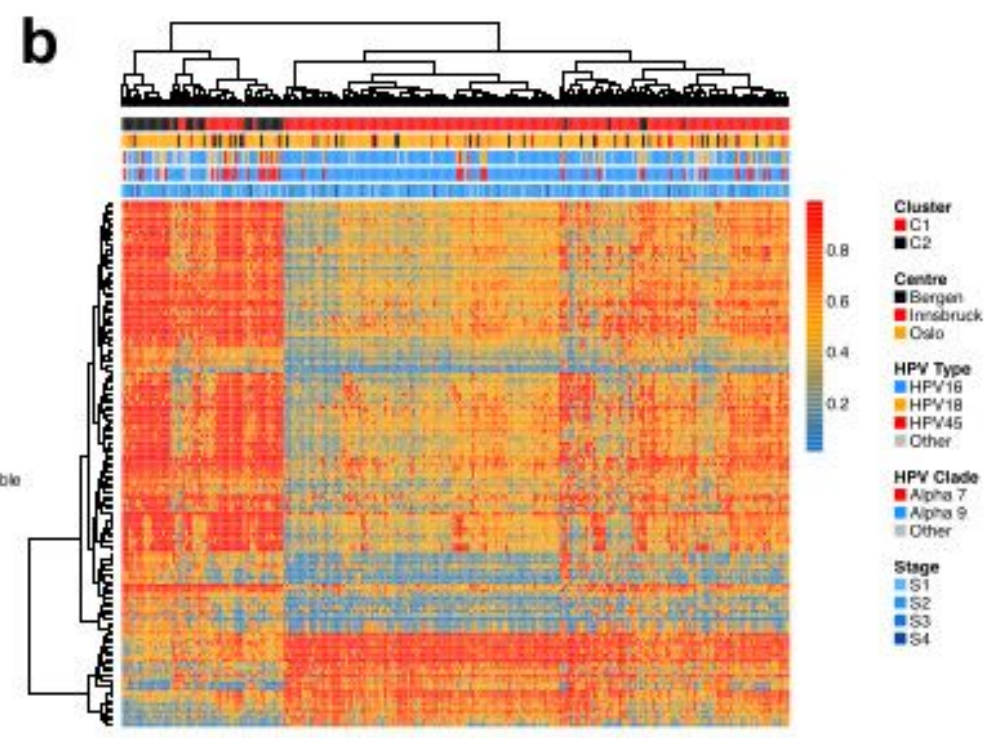

d

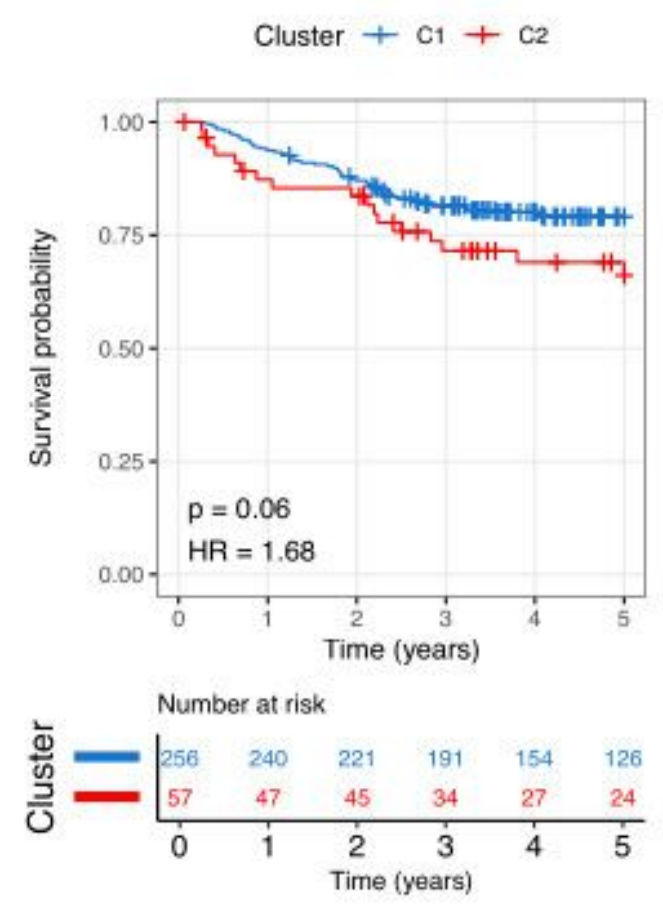

Figure 2 Cluster allocation of validation cohorts using methylation signature. a) A signature of DNA methylation ( $d B>0.25, F D R<0.01$ ) separates $\mathrm{C} 1$ and C2 SCC subgroups in the TCGA cohort. b) The methylation patterns are reproduced in a validation dataset from three European centres $(n=313)$. c) C2 tumours from TCGA and European validation cohorts cluster together based on the 129 MVP signature. d) 5 year survival curve for combined European validation cohorts. Statistics from univariate Cox regression.

\section{Figure 2}

See image above for figure legend. 


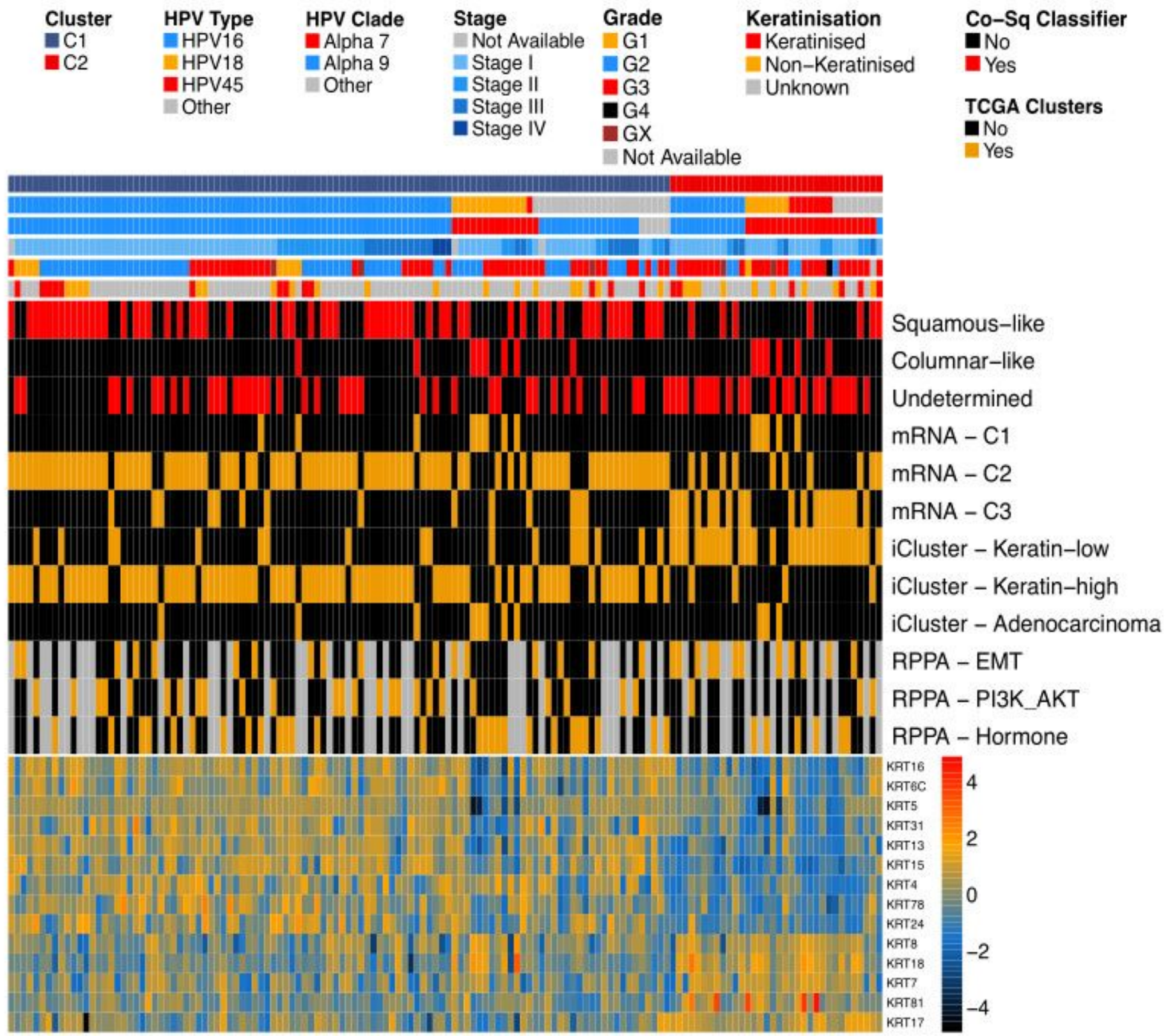

Figure 3 Comparison of SCC subgroups with previous studies. Cluster analysis had previously been performed on 140 TCGA SCC tumours in two studies - one determined clusters based on cell of origin markers (Chumduri et al, 2021, red), one determined clusters based on integrated omics data (TCGA Network, 2017, orange). The heatmap at the bottom of plot represents expression levels of cytokeratin genes present in our C2 gene signature.

Figure 3

See image above for figure legend. 


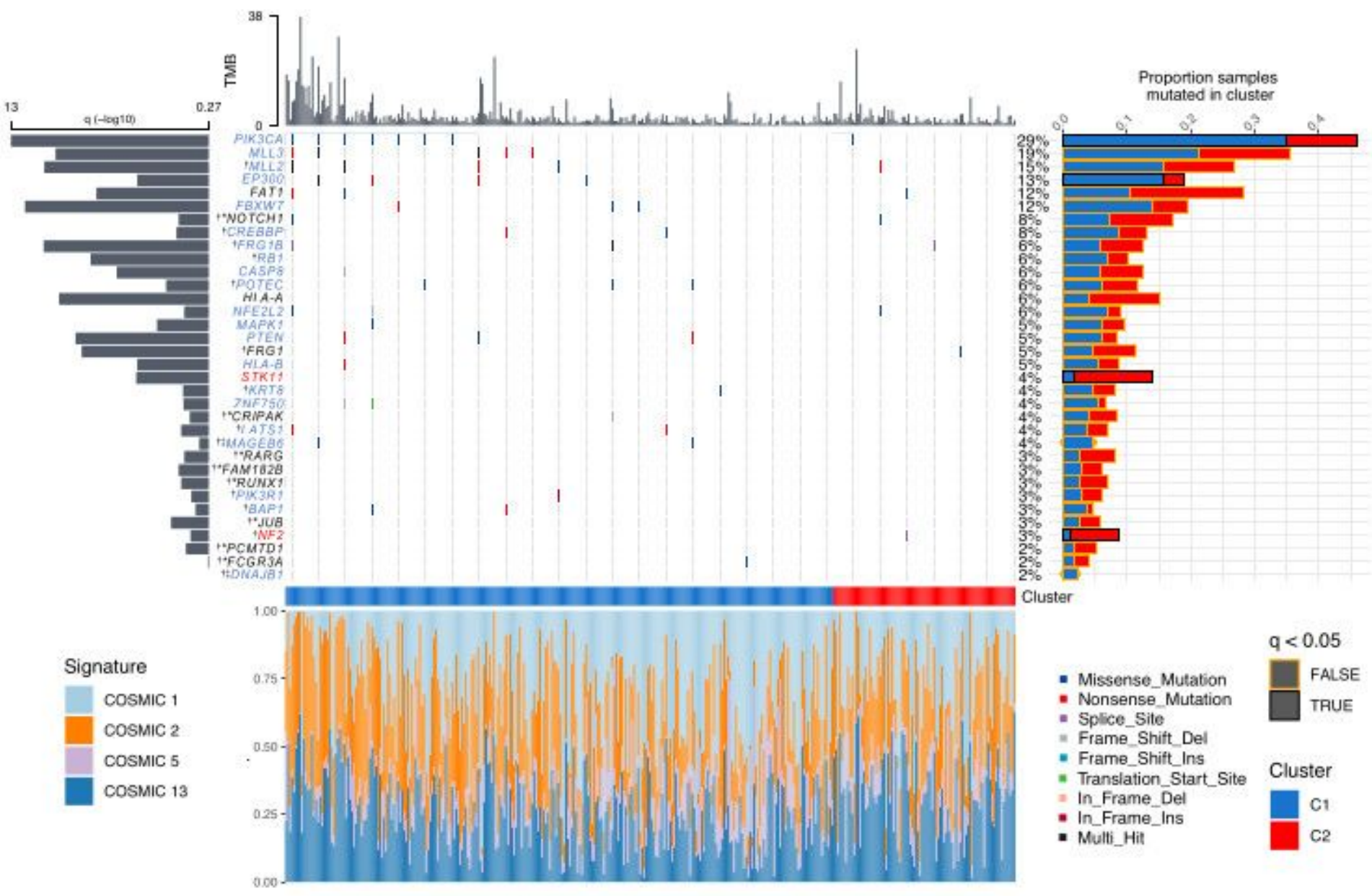

Figure 4 Genomic summary of significantly mutated genes (SMGs) in SCC cohorts. Main plot shows mutation type and frequencies for 34 SMGs identified using dNdSCV on TCGA, Bergen and Ugandan cohorts (367 total patients). Grey bars at top of plot represent TMB per sample. Grey bars to left of plot represent significance of SMG, larger bar is more significant. Barchart to the right shows proportion of a genes mutations in by cluster (blue $=\mathrm{C} 1$, red $=$ C2). Black box around bar represents a significant difference in mutation frequency between the clusters $(p<0.05)$ while a gold box means no significant difference between the clusters. The plot at the bottom of figure represents the mutational signatures that contribute towards each individuals tumour mutational burden.

[Gene name key - blue - unique to $\mathrm{C} 1$ analysis, red = unique to $\mathrm{C} 2$ analysis, black = both in $\mathrm{C} 1$ and $\mathrm{C} 2$ individual analyses, black $^{\star}=$ only significant when combining both clusters for analysis, $\dagger=$ novel SMG in cervical cancer, $\ddagger=$ not significant in combined cluster analysis but significant in $\mathrm{C} 1$ only analysis]

\section{Figure 4}

See image above for figure legend. 
a

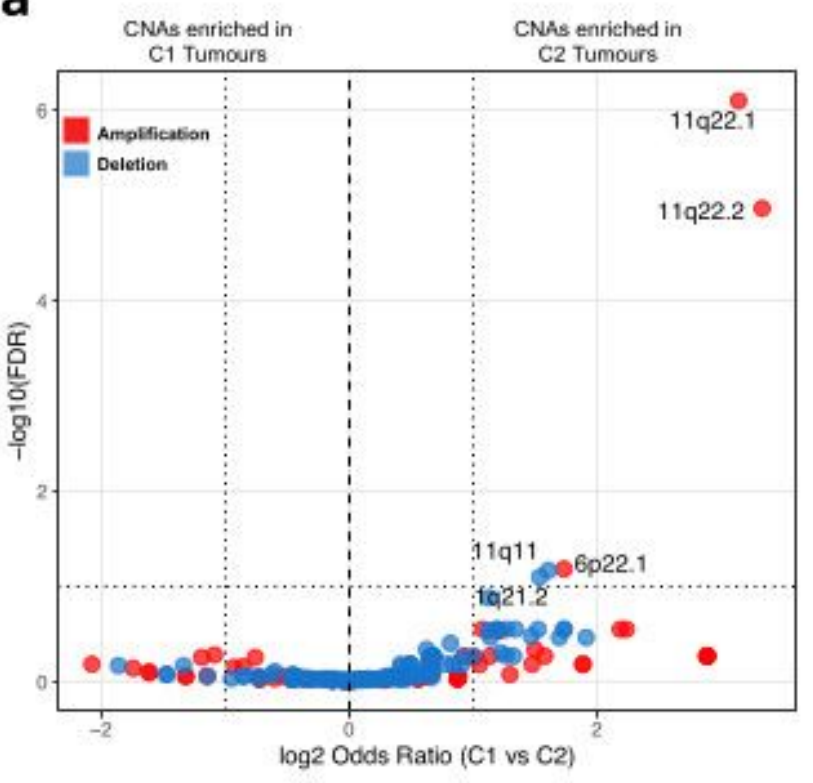

b

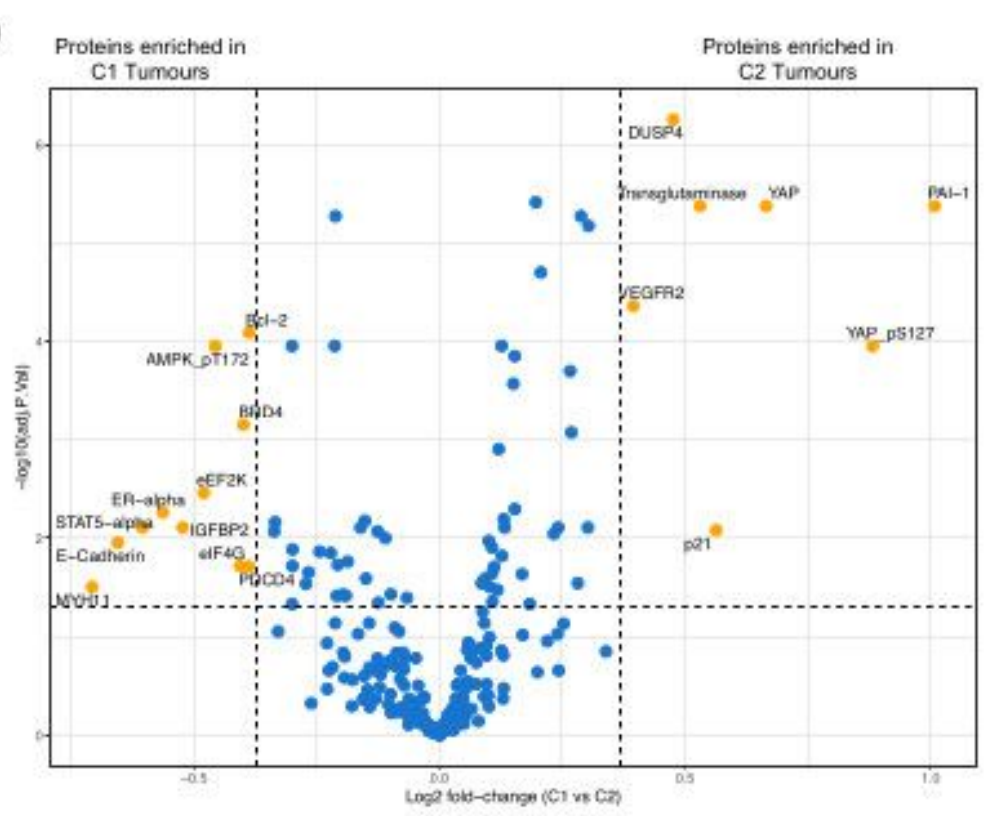

Figure 5 Copy number and protein level differences between SCC subgroups. a) Volcano plot showing differences in GISTIC copy number peak frequencies between $\mathrm{C} 1$ and $\mathrm{C} 2$ tumours, with - $\log 10($ FDR) on the $y$ axis and the odds ratio on the $x$ axis. b) Volcano plot showing differentially abundant proteins and phospho-proteins (FDR < $0.05, F C>1.3$, represented by yellow dots) between C1 and C2 TCGA tumours, as measured by Reverse Phase Protein Array.

\section{Figure 5}

See image above for figure legend. 
a

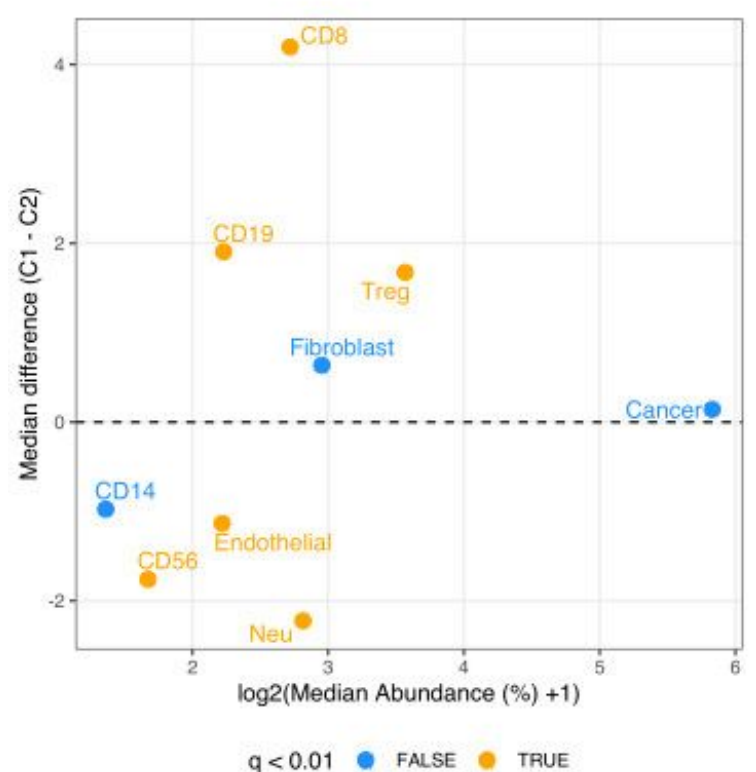

b

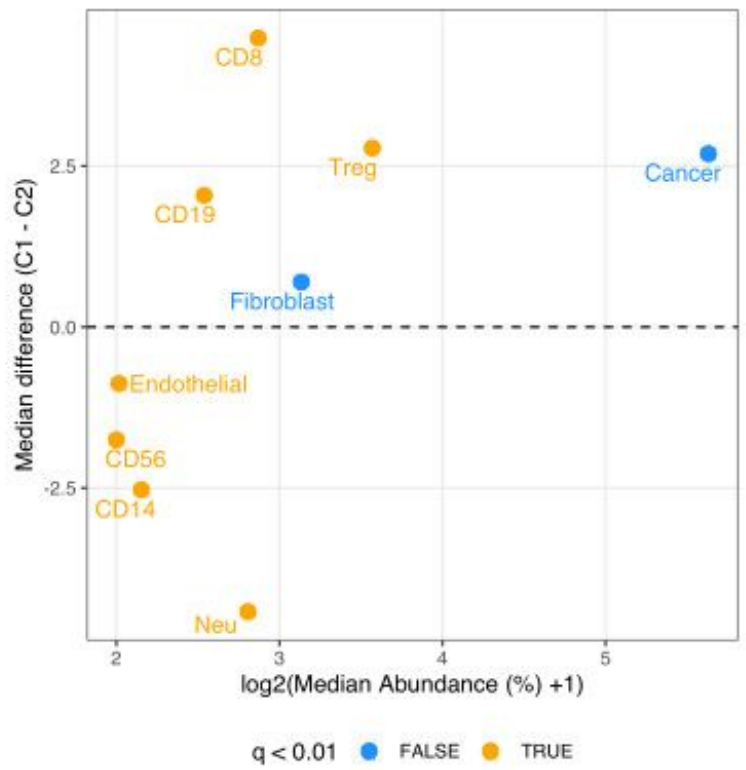

C

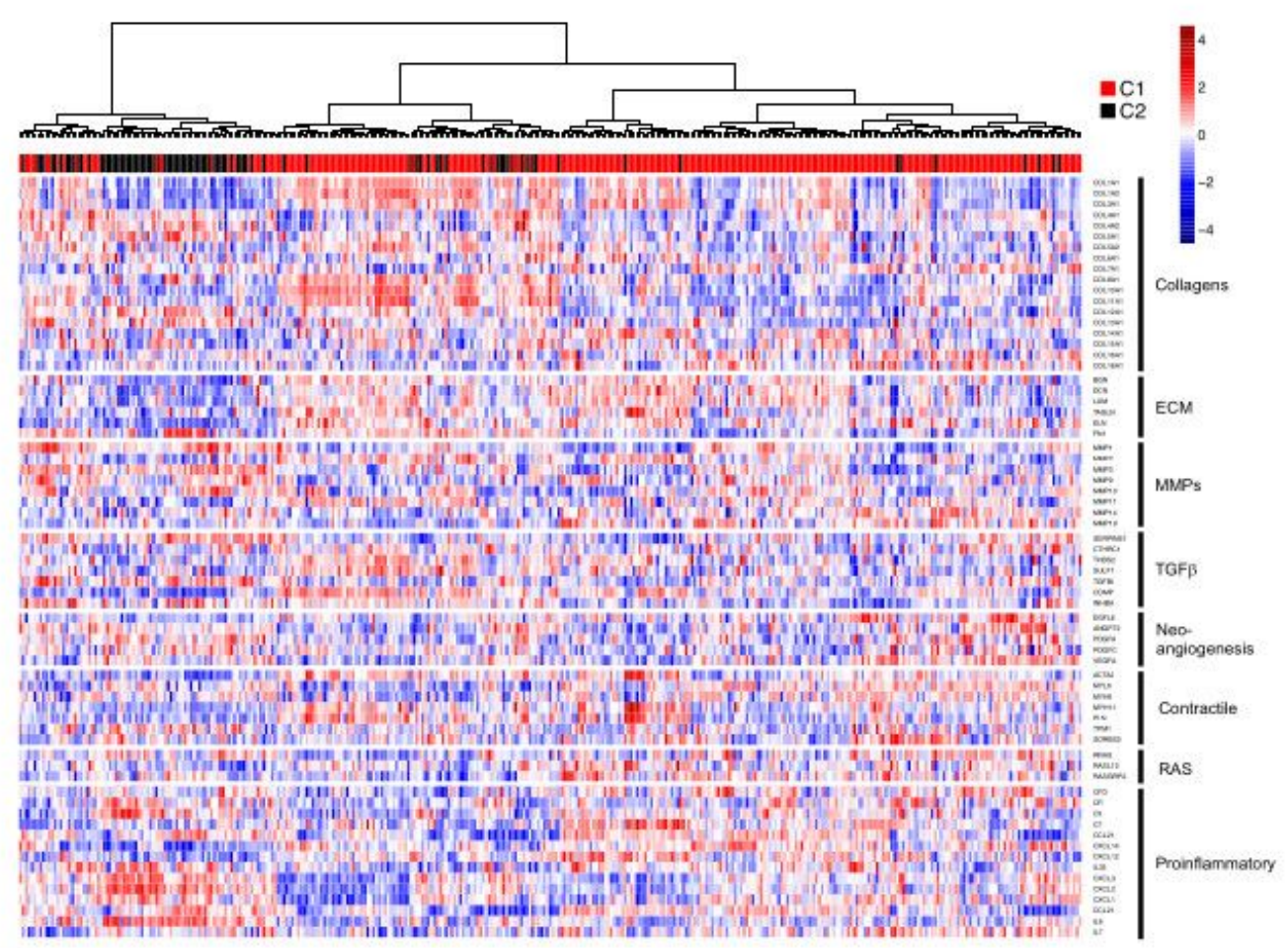

Figure 6 Differences in the tumour microenvironment between cervical cancer subgroups. Plot showing median abundances (x-axis) and median differences (\%,y-axis) for different cell types estimated using MethylCIBERSORT, with significant differences in orange, for a) TCGA discovery cohort and b) combined validation cohorts. c) C2 tumours cluster together using CAF geneset genes.

\section{Figure 6}

See image above for figure legend.

\section{Supplementary Files}

This is a list of supplementary files associated with this preprint. Click to download. 
- suppFig1.pdf

- suppFig2.pdf

- suppFig3.pdf

- suppFig4.pdf

- suppFig5.pdf

- suppFig6.pdf

- suppFig7.pdf

- suppFig8.pdf

- suppFig9.pdf

- suppFig10.pdf

- suppFig11.pdf

- suppFig12.pdf

- supptables.xIsx 